\title{
Neural peptidase endothelin-converting enzyme 1 regulates endothelin 1- induced pruritus
}

\author{
Makiko Kido-Nakahara,, ${ }^{1,2,3}$ Jörg Buddenkotte,,1,2 Cordula Kempkes,,1,4 Akihiko Ikoma, ${ }^{4}$ \\ Ferda Cevikbas, ${ }^{4}$ Tasuku Akiyama, ${ }^{5}$ Frank Nunes, ${ }^{6}$ Stephan Seeliger, 7 Burcu Hasdemir, ${ }^{8}$ \\ Christian Mess, ${ }^{2}$ Timo Buhl,,1,4,9 Mathias Sulk, ${ }^{1,4}$ Frank-Ulrich Müller, ${ }^{6}$ Dieter Metze, ${ }^{2}$ \\ Nigel W. Bunnett, ${ }^{10}$ Aditi Bhargava, 8 Earl Carstens, ${ }^{4}$ Masutaka Furue, ${ }^{3}$ and Martin Steinhoff1,4 \\ 1Department of Dermatology and Charles Institute for Translational Dermatology, University College, Dublin, Ireland. \\ 2Department of Dermatology, University Hospital Münster, Münster, Germany. ${ }^{3}$ Department of Dermatology, Graduate School of Medical Sciences, \\ Kyushu University, Fukuoka, Japan. ${ }^{4}$ Department of Dermatology, UCSF, San Francisco, California, USA. ${ }^{5}$ Department of Neuroscience, UCD, Davis, USA. \\ ${ }^{6}$ Institutes of Pharmacology and Toxicology, University of Münster, Münster, Germany. ${ }^{7}$ Department of Pediatrics III, University of Göttingen, \\ Göttingen, Germany. ${ }^{8}$ Department of Surgery, UCSF, San Francisco, California, USA. 9Department of Dermatology, University of Göttingen, \\ Göttingen, Germany. ${ }^{10}$ Department of Pharmacology and Medicine, Monash University, Victoria, Australia.
}

\begin{abstract}
In humans, pruritus (itch) is a common but poorly understood symptom in numerous skin and systemic diseases. Endothelin 1 (ET-1) evokes histamine-independent pruritus in mammals through activation of its cognate $G$ protein-coupled receptor endothelin A receptor (ETAR). Here, we have identified neural endothelin-converting enzyme 1 (ECE-1) as a key regulator of ET-1-induced pruritus and neural signaling of itch. We show here that ETAR, ET-1, and ECE-1 are expressed and colocalize in murine dorsal root ganglia (DRG) neurons and human skin nerves. In murine DRG neurons, ET-1 induced internalization of ETAR within ECE-1-containing endosomes. ECE-1 inhibition slowed ETAR recycling yet prolonged ET-1-induced activation of ERK1/2, but not $\mathrm{p38}$. In a murine itch model, ET-1-induced scratching behavior was substantially augmented by pharmacological ECE-1 inhibition and abrogated by treatment with an ERK1/2 inhibitor. Using iontophoresis, we demonstrated that ET-1 is a potent, partially histamine-independent pruritogen in humans. Immunohistochemical evaluation of skin from prurigo nodularis patients confirmed an upregulation of the ET-1/ETAR/ECE-1/ERK1/2 axis in patients with chronic $i t c h$. Together, our data identify the neural peptidase ECE-1 as a negative regulator of itch on sensory nerves by directly regulating ET-1-induced pruritus in humans and mice. Furthermore, these results implicate the ET-1/ECE-1/ERK1/2 pathway as a therapeutic target to treat pruritus in humans.
\end{abstract}

\section{Introduction}

Pruritus (itch) is a common symptom of numerous dermatologic, allergic, and autoimmune diseases and tumors, but its molecular and cellular basis is still poorly understood (1). Itch is an unpleasant sensation that elicits the desire to scratch and, as with chronic pain, can be deleterious (1-5). Itch can be triggered by exogenous (e.g., allergens, toxins, medication, illegal drugs, microbes) or endogenous (e.g., amines, proteases, neuropeptides, cytokines, prostanoids) stimuli (4) that transmit signals via $C$ fibers to the central nervous system $(3,6-13)$. While the existence of itchselective $C$ fibers in the sensory nervous system appears to be generally acknowledged $(6,7)$, the neuronal regulatory circuits and the signaling pathways of itch in both the peripheral and central nervous systems, including potential endogenous antipruritic mechanisms, are still mostly unknown (14-16).

Recently, it was discovered that the function of phospholipase C $\beta 3$ (PLC 33 ), a component of a canonical signal transduction cascade, is critical for serotonin- and histamine-induced scratching in mice $(14,17)$. They also reported that histamine requires

Authorship note: Makiko Kido-Nakahara, Jörg Buddenkotte, and Cordula Kempkes contributed equally to this work.

Conflict of interest: The authors have declared that no conflict of interest exists.

Citation for this article: J Clin Invest. 2014;124(6):2683-2695. doi:10.1172/JCI67323. functional transient receptor potential cation channel V1 (TRPV1) to mediate itch transmission, whereas serotonin elicits itch independently of TRPV1 (14). Another TRP family member, TRP ankyrin A1 (TRPA1), is necessary for histamine-independent itch that is induced by Mas-related GPCR-mediated (18) or endothelin 1-induced (ET-1-induced) itch (19). However, ET-1 does not require histamine 1 receptor (H1R), TRPV1, or PLC $\beta 3$ function for itch induction $(14,19)$.

ET-1 is a 21-amino acid peptide and is expressed by a variety of cell types, including immune cells, endothelial cells, neurons, and glial cells of the central and peripheral nervous systems (20-26). ET-1 is a potent vasoconstrictor that can also evoke pain sensations in rodents and humans $(14,22,27-36)$. The biological effects of ET- 1 are mediated by two distinct GPCRs: endothelin A receptor (ETAR) and endothelin B receptor (ETBR) (37). The pruritogenic effect of ET-1 in rodents is mediated in large part by ETAR, although expression of ETBR has been detected in satellite glial cells and nonmyelinated Schwann cells of dorsal root ganglia (DRG) $(33,38)$. When activated, ETAR internalizes and recycles back to the plasma membrane, whereas ETBR internalizes but apparently does not recycle (39). We previously reported that the zinc metalloendopeptidase endothelin-converting enzyme 1 (ECE-1) is present in acidified endosomes and degrades neuropeptides to promote recycling and resensitization of GPCRs that induce 


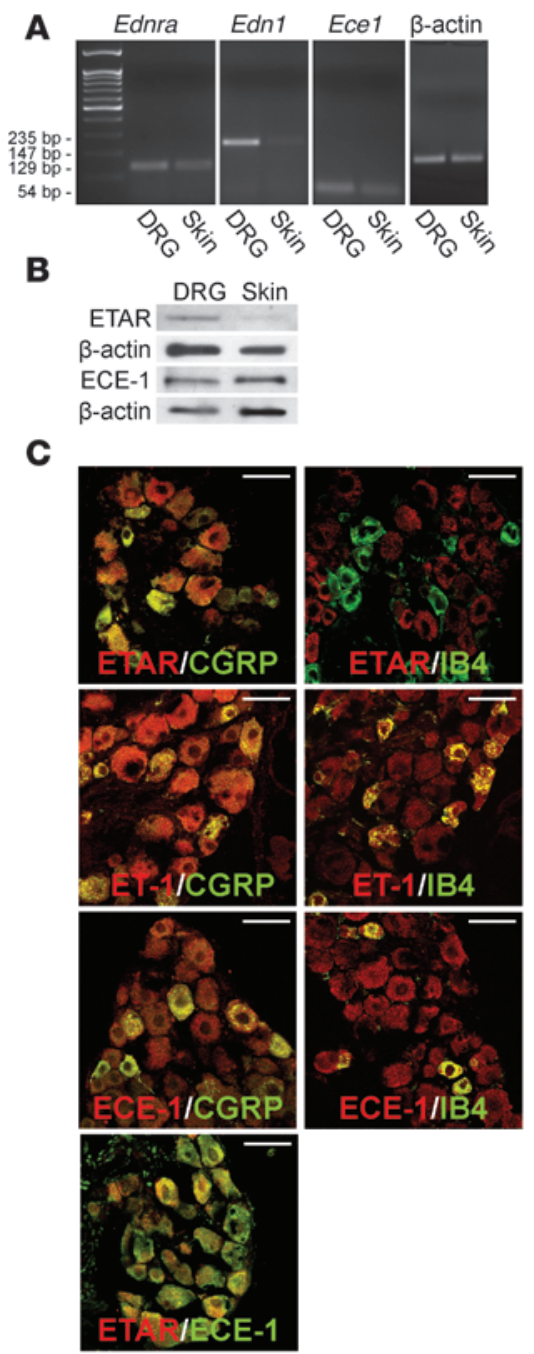

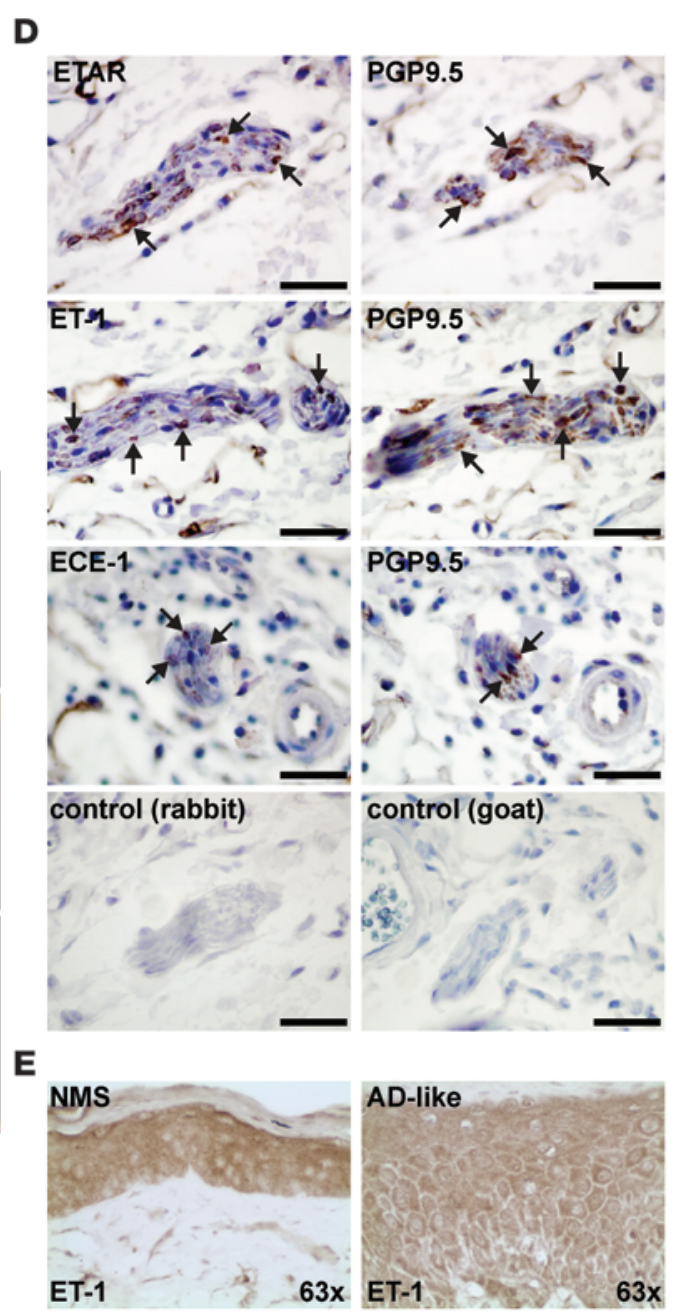

Figure 1

ETAR, ET-1, and ECE-1 are expressed in adult mouse DRG neurons and skin. (A) Ednra, Edn1, and Ece1 mRNAs were detectable in mouse DRG neurons and skin. (B) Expression of ETAR and ECE-1 protein was confirmed for mouse DRG neurons and skin by Western blot analysis. (C) ETAR, ET-1, and ECE-1 were detected by IHC in small- to medium-sized DRG neurons and colocalized to some extent with CGRP, IB4, and TRPV1 (arrows, see Table 1). Scale bars: $50 \mu \mathrm{m}$. (D) ETAR, ET-1, and ECE-1 colocalized with PGP9.5+ peripheral sensory nerves (arrows) of skin, as examined by IHC using serial sections of regional skin obtained from mice suffering from chronic pruritic dermatitis. Scale bars: $50 \mathrm{um}$. (E) ET-1 expression in epidermal layers of biopsies obtained from WT mice (NMS, normal mouse skin) or mice with an AD-like phenotype visualized with IHC. Original magnification, $\times 63$.

neurogenic inflammation (40-42) and to terminate endosomal neuropeptide signaling $(40,41,43)$. Based on our previous studies showing that ECE-1 regulates neuropeptide function, we hypothesized that neural ECE-1 plays a fundamental role in regulating ET-1-induced itch.

Our results indicate that endosomal ECE- 1 modulates itch by regulating ET-1 and extracellular signal-regulated kinase 1/2 (ERK1/2) signaling in mice and appears to be the first identified endogenous negative regulator of itch signaling in sensory nerves. In a translational setting, we also demonstrate a role of ET-1 in human itch that may lead to specific treatments for this difficult field of therapeutic medicine.

\section{Results}

ETAR, ET-1, and ECE-1 colocalize in murine dorsal root ganglion neurons and skin. Recently, we have demonstrated that ECE-1 is an important regulator of neuropeptide-induced skin inflammation (40). Because ECE-1 also regulates ET-1 function (44), we tested the hypothesis that ECE-1 contributes to ET-1-mediated itch behavior in mice. We first determined the expression of ET-1, ETAR, and ECE-1 in skin, cutaneous nerve fibers, and DRG neurons. RT-PCR confirmed the presence of $E d n 1, E d n r a$, and Ece1 mRNA in skin and the peripheral nervous system (Figure 1A). Protein expression of ETAR and ECE- 1 by DRG neurons and skin was also confirmed by Western blot analysis (Figure 1B). We found that immunoreactive ET-1, ETAR, and ECE-1 were mainly localized in a subset of small- to medium-sized DRG neurons, where $95.0 \pm 1.4 \%$ of ETARpositive neurons expressed ECE-1 (Figure $1 \mathrm{C})$. Further, we classified these neurons on the basis of size (Table 1). Of the ETAR-expressing neurons, $69.2 \pm 2.0 \%$ were small-sized (diameter $<25 \mu \mathrm{m})$ and $30.8 \pm 2.0 \%$ were medium-sized (25-40 $\mu \mathrm{m})$. Of the ET-1positive DRG neurons, $82.0 \pm 2.8 \%$ were small-sized DRG neurons and $18.0 \pm 2.5 \%$ were medium-sized. For all ECE-1-positive neurons, $70.1 \pm 3.6 \%$ were small-sized, $28.7 \pm 3.4 \%$ were medium-sized, and $1.2 \pm 0.2 \%$ were large-sized (diameter $>40 \mu \mathrm{m}$ ) (Table 1 ). ETAR, ET-1, and ECE-1 colocalized with the peptidergic marker calcitonin gene-related peptide (CGRP) (ETAR, $37.5 \pm 0.7 \%$; ET-1, $40.6 \pm 5.1 \%$; ECE- 1 , $39.0 \pm 4.7 \%)$ and to a lesser extent with the nonpeptidergic marker isolectin B4 (IB4) (ETAR, $7.9 \pm 3.5 \%$; ET-1, $42.1 \pm 1.1 \%$; ECE-1, $26.1 \pm 5.3 \%$ ) (Figure 1C and Table 1). Furthermore, 33\% of ETAR- and ET-1-positive neurons also expressed TRPV1, which is generally expressed by sensory nerves conveying itch as well as pain signals $(14,45,46)$.

We examined the cutaneous expression of ET-1, ETAR, and ECE-1 in mice with pruritic chronic dermatitis and observed staining for ET-1, ETAR, and ECE-1 in keratinocytes, inflammatory cells, and endothelial cells; no staining was discernible in fibroblasts (data not shown) in any of the analyzed skin samples. ET-1, ETAR, and ECE-1 also colocalized with the neuronal marker protein gene product 9.5 (PGP9.5) in subepidermal cutaneous nerve fibers (Figure 1D). Induction of an atopic-like phenotype induced increased ET-1 staining in the epidermal layer compared with that seen in normal murine skin (Figure 1E). 
Table 1

Morphometric analysis of ETAR-, ET-1-, and ECE-1-positive murine DRG neurons

\begin{tabular}{|c|c|c|c|c|c|}
\hline & $\begin{array}{c}\text { Small-sized } \\
(<25 \mu \mathrm{m})\end{array}$ & $\begin{array}{l}\text { Medium-sized } \\
(25-40 \mu \mathrm{m})\end{array}$ & $\begin{array}{l}\text { Large-sized } \\
(>40 \mu \mathrm{m})\end{array}$ & CGRP+ & IB4+ \\
\hline & $69.2 \pm 2.0$ & $30.8 \pm 2.0$ & - & $37.5 \pm 0.7$ & \\
\hline (1) & $82.0 \pm$ & $18.0 \pm$ & - & $40.6 \pm 5.1$ & $42.1 \pm 1.1$ \\
\hline E-1+ neurons & $70.1 \pm 3.6$ & $28.7 \pm 3.4$ & $1.2 \pm 0.2$ & $39.0 \pm 4.7$ & $26.1 \pm 5.3$ \\
\hline
\end{tabular}

Values are expressed as the mean \pm SEM $(\%) ; n=3$. -, no expression detected.

tion by a functional H1R (Figure 2F). Also Trpv1-/, $\operatorname{Trp} v 4^{4^{--}}$, and $\operatorname{Trpm} 8^{-{ }^{--}}$mice showed no differences in ET-1-induced scratching responses (Figure 2A), whereas $\operatorname{Trpa1}^{-/-}$mice showed a marked reduction in ET-1-triggered itch when compared with WT mice (Figure 2A). Therefore, we i.p. injected a TRPA1 inhibitor $(30 \mathrm{mg} / \mathrm{kg}, \mathrm{HC}-030031) 30 \mathrm{~min}-$ utes before ET-1 injection (100 pmol/site) into the cheek and observed reduced scratching behavior as compared with vehicle control (Supplemental Figure 3). Because ETAR, ET-1, and ECE-1 are colocalized in neuronal cells that belong to the

ET-1 induces strong $\mathrm{Ca}^{2+}$ signals in neurons (47) and epithelial cells (48). To investigate the functional expression of ET-1, ETAR, and ECE- 1 in sensory nerves, we examined the effects of ET-1 on the modulation of $\left[\mathrm{Ca}^{2+}\right]_{\mathrm{i}}$ in murine $\mathrm{DRG}$ neurons in vitro. Stimulation with $100 \mathrm{nM}$ ET-1 increased $\left[\mathrm{Ca}^{2+}\right]_{\mathrm{i}}$ in DRG neurons (Supplemental Figure 1; supplemental material available online with this article; doi:10.1172/JCI67323DS1), which indicates that ET-1 activates intracellular signaling cascades in murine DRG neurons. Based on the expression pattern of ETAR and ETBR in murine DRG neurons, ET-1 most likely exerts its actions on small- to medium-sized sensory nerves by binding to ETAR. As with the percentage of neurons that respond to other endogenous pruritogens, such as bovine adrenal medulla 8-22 peptide (BAM8-22) or thymic stromal lymphopoietin $(49,50)$, only a small proportion of these small- to medium-sized DRG neurons (3.0\%; $78 / 2589)$ exhibited a transient increase in $\left[\mathrm{Ca}^{2+}\right]_{\mathrm{i}}$ in response to ET-1 application (Figure 2B). We found that $69.2 \%$ of 13 ET-1responsive DRG neurons responded to histamine, $24.0 \%$ of 25 cells responded to chloroquine (CQ), $14.8 \%$ of 27 cells responded to SLIGRL-NH $\mathrm{N}_{2}, 85.5$ of 29 cells responded to capsaicin, and $73.5 \%$ of 49 cells responded to allyl isothiocyanate (AITC, mustard oil) (Figure 2, C and D). To further examine the role of TRPV1 and TRPA1 for ET-1-evoked $\left[\mathrm{Ca}^{2+}\right]$ i, we additionally tested $\operatorname{Trpa1}^{-/-}$and

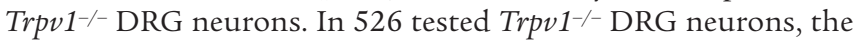
percentage of ET-1-responsive DRG neurons was comparable to that in WT DRG neurons (Figure 2B). The percentage of ET-1responsive Trpa1 ${ }^{-1-}$ DRG neurons tended to be reduced slightly, but was not significant in 901 tested cells (Mann-Whitney $U$ test, $P=0.595$; Figure 2B). Our data suggest that ET- 1 does not activate a subset of TRPV1-positive sensory neurons, but may act partly via TRPA1-positive sensory neurons. Our results also indicate that ET-1, ETAR, and ECE-1 are expressed by small- to medium-sized sensory nerves projecting into the skin and suggest that ET-1/ ETAR-induced itch may be modulated by endosomal ECE- 1 in peripheral sensory neurons in mice.

Inbibition of ECE-1 enhances ET-1-induced scratching behavior in vivo. ET-1 evokes itch sensation in BALB/c and male Swiss mice $(27,33)$. Intradermal (i.d.) injection of ET-1 (100 pmol, and 10 pmol, respectively) into the cheek also evoked a marked scratching response in C57BL/6 WT mice (Figure 2, A and E, and refs. 51, 52). Gomes et al. (51) reported that ET-1 also acts as a nociceptive agonist, thus we took advantage of the cheek model to distinguish between ET-1induced scratching and pain behavior (53). In our experimental setting, ET-1 induced a concentration-dependent scratching response (from $1 \mathrm{pmol}$ to $1 \mathrm{nmol} / \mathrm{site}$, Supplemental Figure 2). However, in contrast to the published observation, we did not observe any wiping movements in any treated mouse using those concentrations (data not shown). ET-1-induced itch was independent of a contribu-
C fiber class of neurons, we hypothesized that ECE-1 controls ET-1-evoked itch. To examine this possibility, we pretreated mice with SM-19712, a specific ECE-1 inhibitor, followed by i.d. injection of ET-1 (100 pmol) into the cheek, and measured scratching behavior. We found a significant increase in scratching bouts when ET-1 was applied after SM-19712 pretreatment (ECE-1 blockage). In contrast, SM-19712 pretreatment alone did not change scratching behavior in mice in vivo (data not shown).

ET-1 induces internalization of ETAR in DRG neurons. To determine whether ET-1 stimulates activation and trafficking of ETAR, we examined the subcellular localization of immunoreactive ETAR and ECE-1 in DRG neurons by confocal microscopy. In unstimulated small-diameter DRG neurons, we found that ETAR was prominently localized at the cell surface, whereas ECE-1 was mainly localized in vesicles (Figure 3A).

We have previously shown in KNRK and HEK cells that ECE-1b and ECE-1d isoforms are colocalized with early endosomal antigen 1 (EEA-1) in endosomes (40). Previous studies with Chinese hamster ovary $(\mathrm{CHO})$ cells demonstrated that ET-1 colocalizes with ETAR in internalized vesicular structures $(39,54)$. When DRG neurons were stimulated with ET-1 (100 nM) for $10 \mathrm{~min}$ utes, ETAR redistributed to early endosomes containing ECE-1 (Figure 3A) and EEA-1 (Supplemental Figure 4). Using ImageJ quantification, we demonstrated that immunofluorescence for ETAR decreased on the cell membrane and increased in the cytosol after ET-1 stimulation (Figure 3C). ETAR reappeared on the cell membrane 60 minutes after ET-1 stimulation, whereas ECE-1 relocalized in ETAR-negative endosomes, indicating receptor recycling (Figure 3A, Figure 3C for statistical analysis, and ref. 40).

We also confirmed that internalized ET-1 colocalized with ECE-1 (Supplemental Figure 5) and EEA-1 (Supplemental Figure 8). Immunofluorescence for ET-1 increased after ET-1 stimulation but disappeared over time, whereas ECE-1 immunofluorescence was constant over a 120-minute time course (Supplemental Figure 9). Therefore, stimulation of ETAR on DRG neurons by ET-1 induces colocalization of the ligand/receptor complex in early endosomes, which are also ECE-1 positive. Our results indicate that ETAR recycles back to the cell membrane, while ET-1 is possibly degraded.

We determined the role of ECE-1 in ETAR recycling. Pretreatment of cultured primary DRG neurons with the ECE- 1 inhibitor SM-19712 $(10 \mu \mathrm{M})$ did not influence ET-1-evoked internalization and colocalization of ETAR with ECE-1, but prolonged colocalization of ETAR with ECE-1 in endosomes and prevented recycling of the receptor back to the cell surface even after 60 minutes (Figure 3, $B$ and C). Previous studies in KNRK and HEK model cell lines have shown that ECE-1 degrades neuropeptides in acidified early endosomes and allows the receptor, freed from agonist and $\beta$-arrestins, to recycle back to the plasma membrane $(40,42)$. Here, we show for 
A

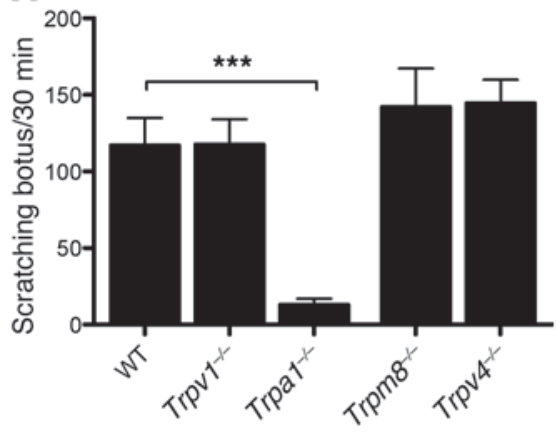

D

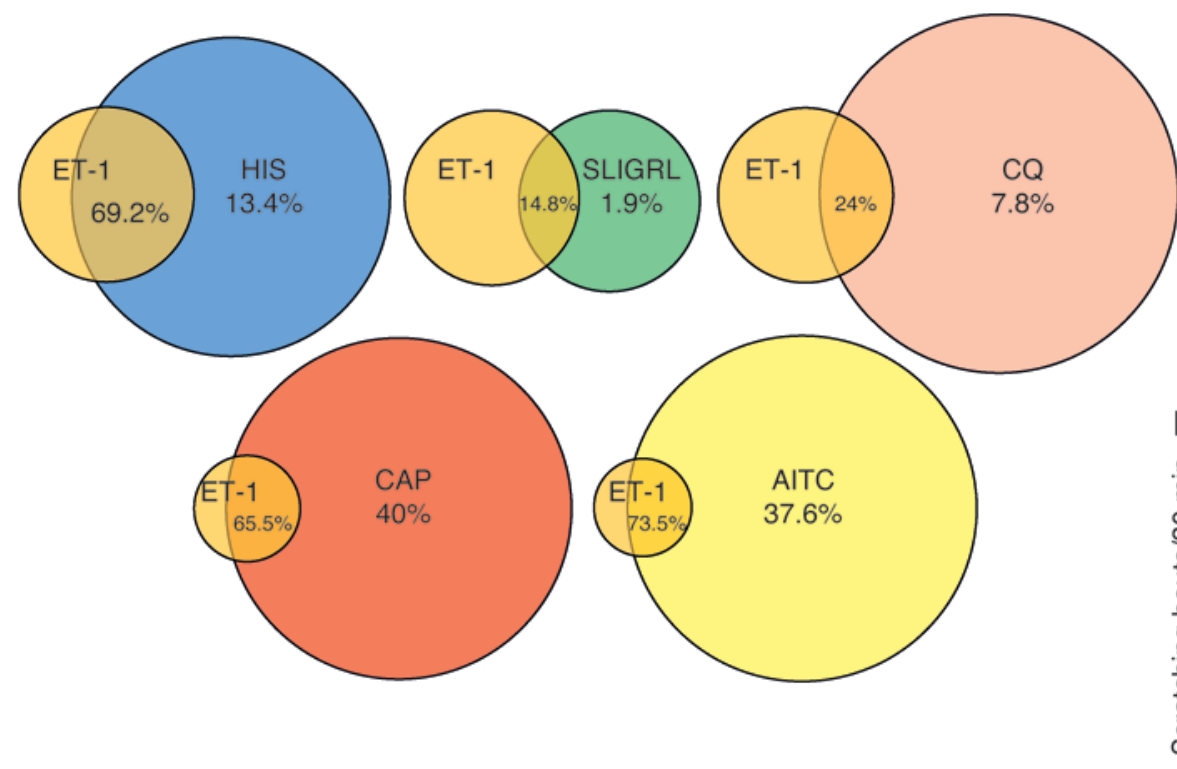

B

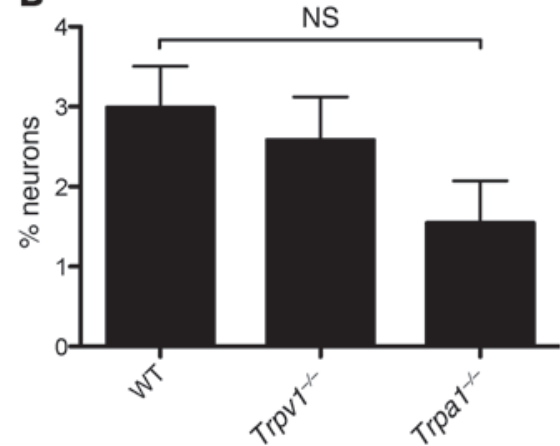

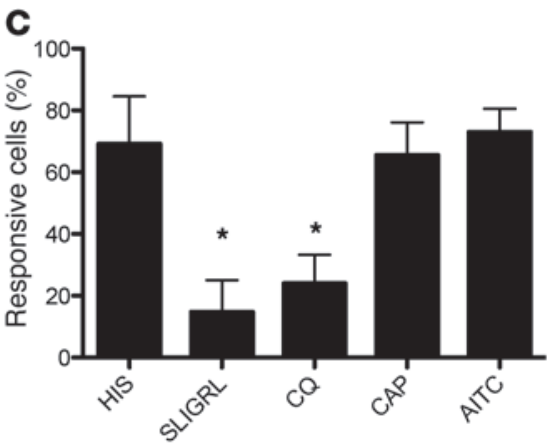

$\mathbf{E}$

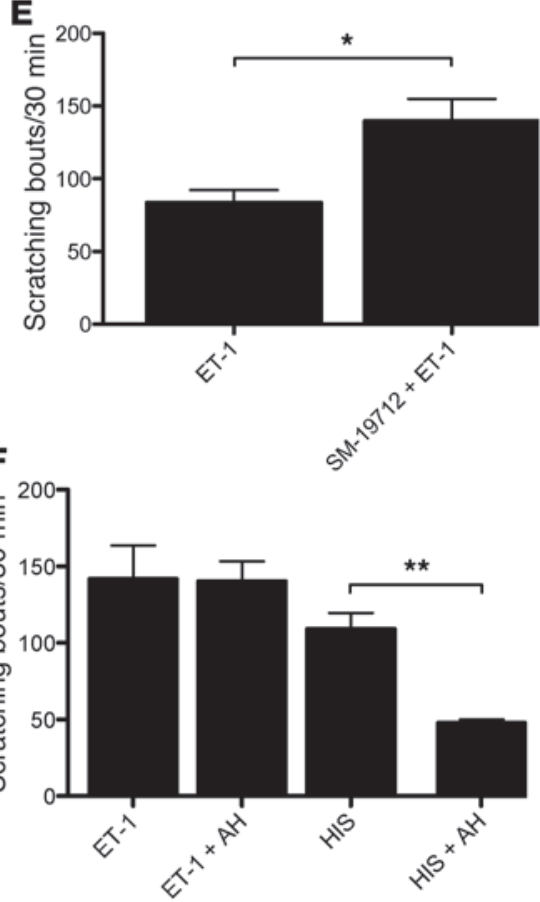

Figure 2

ET-1-induced scratching behavior in mice is dependent on TRPA1 and ECE-1, but not H1R, activation. (A) Scratching elicited by ET-1 (100 pmol/10 $\mu$ linjected into the cheek) was eliminated in Trpa1-deficient mice but not in Trpv1-, Trpv4-, or Trpm8-deficient mice. Total number of scratching bouts per 30 minutes ( $n=4-10$ mice/group). (B) ET-1-induced calcium mobilization. Percentage of ET-1-responsive neurons that also responded to other compounds (Mann-Whitney $U$ test, $n=409-1,525$ cells/group). For quantification, 15-68 dishes per group were used, and approximately 40 cells per dish were counted. (C) Characterization of ET-1-responsive DRG neurons. Percentage of ET-1-responsive neurons in different $\mathrm{KO}$ mice (Mann-Whitney $U$ test, $P<0.05 ; n=526-1,057$ cells/group) compared with either the histamine (HIS), capsaicin (CAP), or mustard oil (AITC) group. Error bars indicate the SEM. (D) Venn diagrams showing percentages of DRG neurons. (E) ECE-1 modulated itch behavior responses to ET-1. Total number of scratching bouts over the 30-minute observation period showed substantial enhancement in behavioral response to i.d. administered ET-1 (cheek; $n=5,10 \mathrm{pmol} / \mathrm{site}$ ) when mice were pretreated with ECE-1 inhibitor SM-19712 thirty minutes prior to agonist administration $(n=7 ; 25 \mathrm{mg} / \mathrm{kg}$,). At this concentration, no pain behavior was observed. (F) ET-1-elicited scratching behavior was independent of functional $\mathrm{H} 1 \mathrm{R}$. Diphenhydramine hydrochloride (antihistamine $[\mathrm{AH}] ; 3 \mathrm{mg} / \mathrm{kg}$ in $\mathrm{H}_{2} \mathrm{O}$ ) was intragastrically administered 30 minutes prior to i.d. ET-1 injection (100 pmol/100 $\mu$; nape of the neck; $\left.n=4-5) .{ }^{\star} P<0.05 ;{ }^{\star \star} P<0.01 ;{ }^{* \star} P<0.001\right)$.

the first time, to the best of our knowledge, that ECE-1 has a similar role in DRG neurons, which naturally express ET-1 and ETAR. Internalized ET-1 is degraded by endosomal ECE-1. It is well known that extracellular localized ECE-1 hydrolyzes big endothelin 1 at a neutral $\mathrm{pH}$ and thereby generates bioactive ET-1 (44). However, endosomal ECE-1 isoforms can degrade neuropeptides in an acidic milieu with an optimum pH of 5.6 to 5.8 (44). Our results show that ECE- 1 colocalized with ET-1 and ETAR in endosomes of DRG neurons (Figure 3 and Supplemental Figures 4, 5, and 8).
These data support the hypothesis that ECE-1 degrades ET-1 in acidified endosomes of DRG neurons to regulate ETAR recycling and endosomal signaling.

To test this hypothesis, we examined ECE-1-mediated degradation of ET-1 at an acidic endosomal $\mathrm{pH}$ using an in vitro approach; ET-1 was incubated with recombinant human ECE-1 (rhECE-1) at endosomal ( $\mathrm{pH} 5.5)$ and extracellular ( $\mathrm{pH} 7.4)$ acidities for 90 minutes. We analyzed the degradation products by HPLC and matrix-assisted laser desorption/ionization time-of-flight 
A
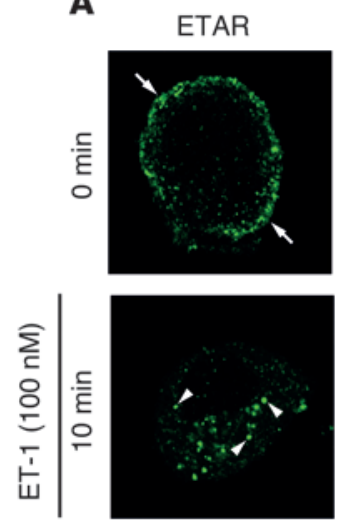

Wash

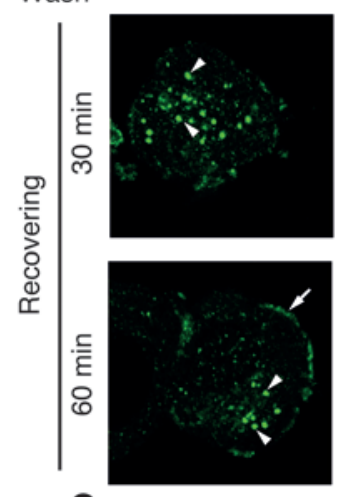

C
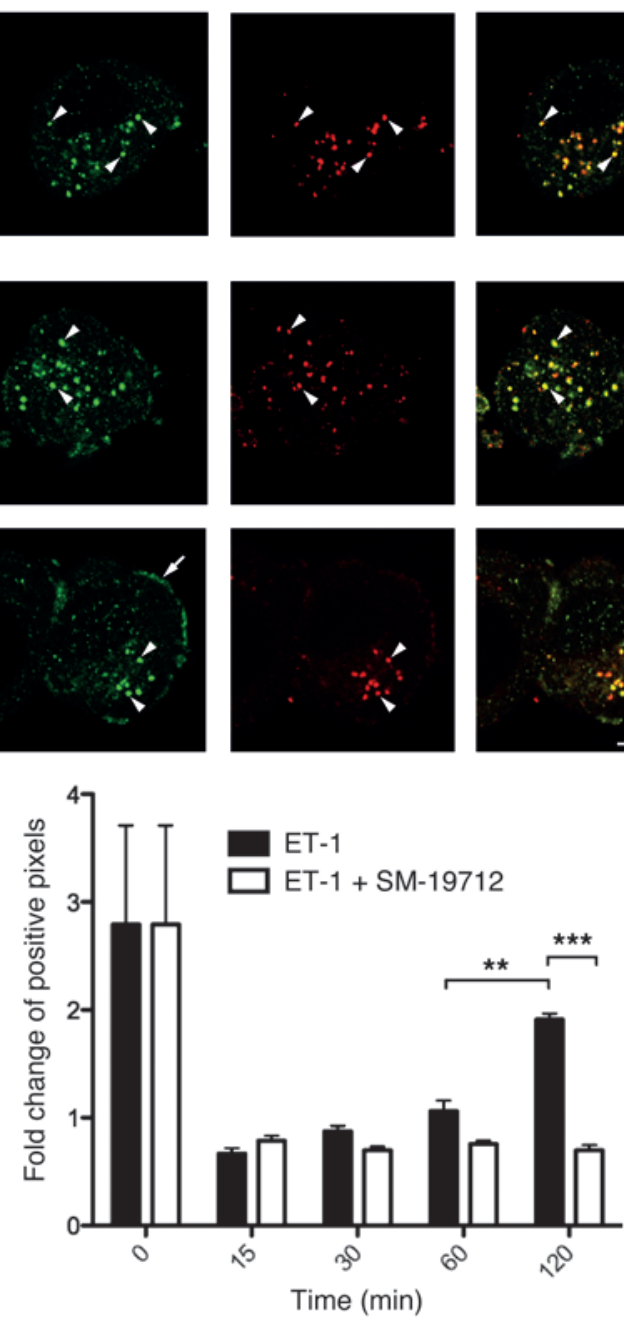

B
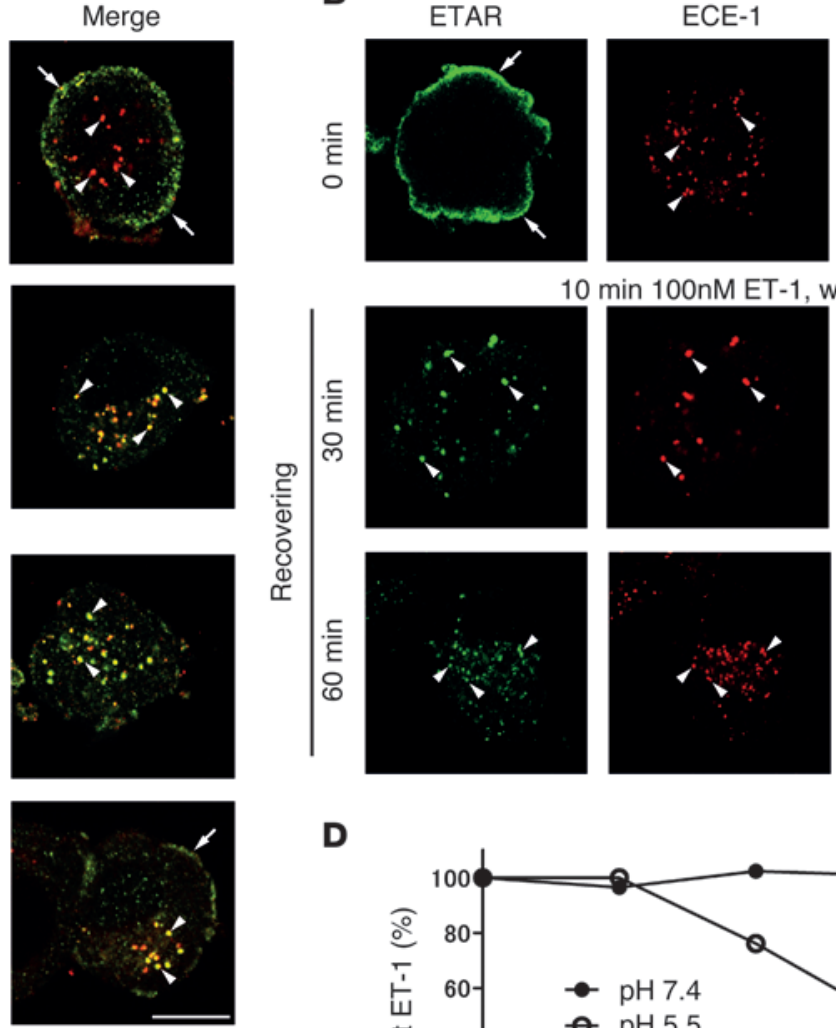

D

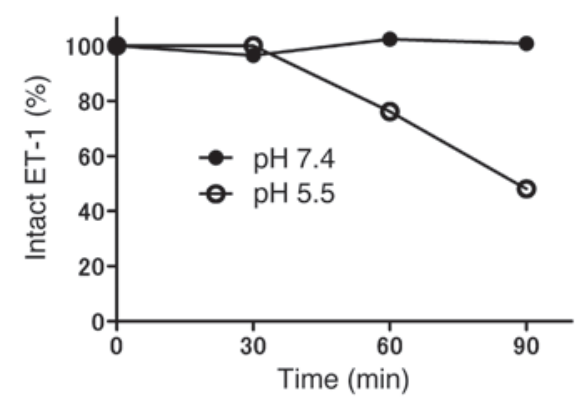

$\mathbf{E}$

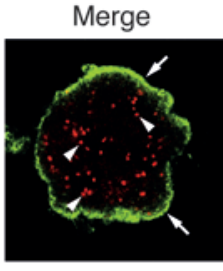

10 min 100nM ET-1, wash
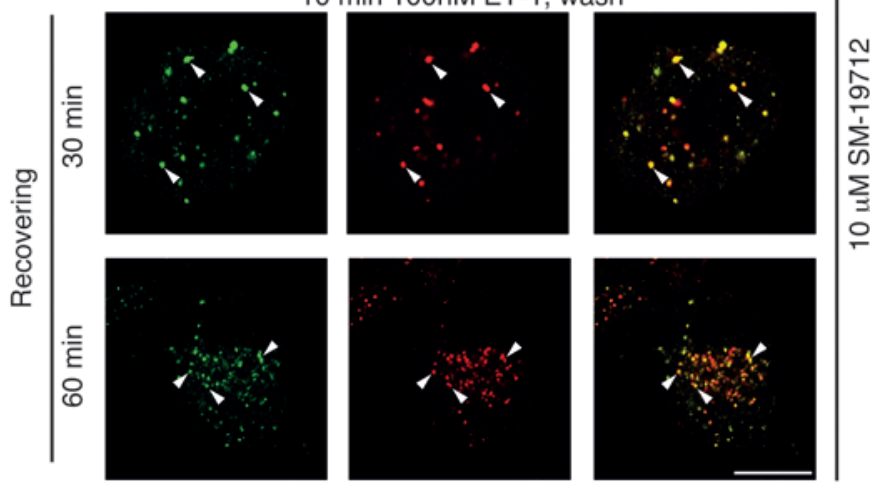

D

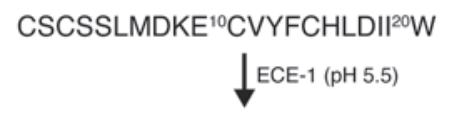

CSCSSLMDKE ${ }^{10} \mathrm{CVYFCHLD} \mathrm{+} \mathrm{IIW}$

Figure 3

ECE-1 is important for the recycling of ETAR in DRG neurons. DRG neurons were cultured with ET-1 in the presence or absence of ECE-1 inhibitor. (A) Membrane-bound ETAR (arrows) internalized and colocalized with ECE-1 (arrowheads) in cytosolic vesicles of DRG neurons within 10 minutes after ET-1 stimulation. Recovery of the receptor on the plasma membrane was observed 60 minutes after ET-1 application (arrow). Scale bar: 30 um. (B) ECE-1 inhibitor SM-19712 did not affect initial internalization of ETAR (arrows, arrowheads), but prevented recycling of ETAR to the plasma membrane. Scale bar: $30 \mu \mathrm{m}$. (C) Time dependence of ETAR translocation in DRG neurons in response to ET-1 $(100 \mathrm{nM})$. In unstimulated cells, ETAR was mainly located at the plasma membrane. ET-1 stimulation for 10 minutes induced translocation of ETAR from the plasma membrane to the cytosol. Immunofluorescence for ETAR reappeared at the plasma membrane 60 minutes after stimulation (black bars). Costimulation with the ECE-1 inhibitor SM-19712 (10 $\mu \mathrm{M})$ inhibited relocation of ETAR immunofluorescence to the plasma membrane ( $n=10$ cells/group, error bars indicate \pm SEM, Student's $t$ test, ${ }^{* \star} P<0.01$; $\left.{ }^{* \star} P<0.001\right)$. Subcellular distribution was analyzed from captured images using ImageJ software. (D) ECE-1 degraded ET-1 at endosomal pH 5.5, but not at pH 7.4. Degradation of ET-1 by rhECE-1 at $\mathrm{pH} 5.5$ and $\mathrm{pH} 7.4$ was assessed by HPLC. (E) ET-1 was hydrolyzed by ECE-1 at Asp ${ }^{18}-$ Ile $^{19}$. 


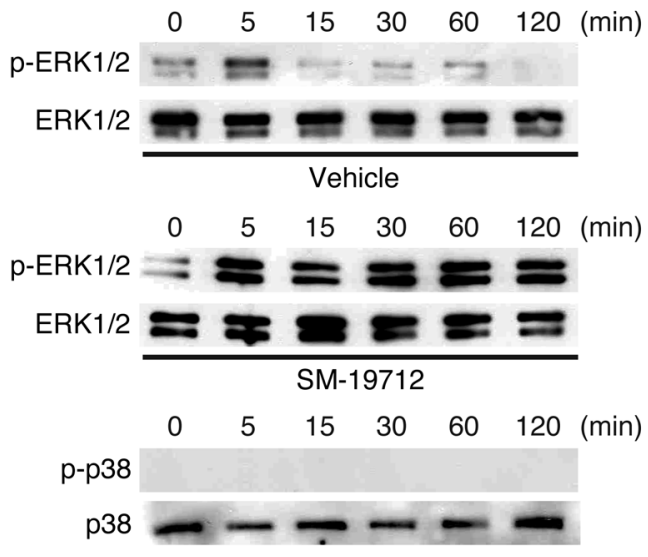

\section{Figure 4}

ECE-1 inhibition prolongs ET-1-induced phosphorylation of ERK1/2 in DRG neurons. DRG cells were pretreated with SM-19712 (10 $\mu \mathrm{M})$ or vehicle for 60 minutes and stimulated with ET-1 (100 nM). The cellular lysates were subjected to Western blot analysis for ERK1/2 and p38. ET-1 caused ERK activation in DRG neurons, reaching a maximum after 5 minutes, and ERK activation was sustained by pretreatment with SM-19712. ET-1 did not activate p38 in DRG neurons. Data are representative of three independent experiments.

mass spectrometry (MALDI-TOF). HPLC showed that intact ET-1 control peptide that was not incubated with ECE-1 was eluted at 28 minutes. After incubation with rhECE- 1 at $\mathrm{pH} 5.5$, two degradation products that eluted at 24 and 26 minutes were identified by HPLC. During incubation for 90 minutes, intact ET-1 decreased to $48 \%$ of the initial value, and the two degradation products increased (Figure 3D and Supplemental Figure 6). Mass spectrometric analysis indicated that ET-1 was hydrolyzed by ECE-1 between amino acid residues Asp ${ }^{18}$ Ile $^{19}$ (Figure 3E). ET-1 degradation was $\mathrm{pH}$ dependent, since there was minimal degradation at pH 7.4 (99\% intact ET-1 after a 90-minute incubation with rhECE-1). Together, these results indicate that internalized ET-1 could be degraded by endosomal ECE-1 at an acidic $\mathrm{pH}$ in DRG neurons in vivo.

ECE-1 inbibitor prolongs ET-1-induced phosphorylation of ERK1/2 in $D R G$ neurons in vitro. Our results clearly demonstrate that ECE-1 promotes ETAR recycling, and thus resensitization, in DRG neurons in vitro and that inhibition of ECE-1 intensifies ET-1-evoked scratching behavior in mice in vivo. These observations indicate a fundamental contribution of ligand (ET-1) dissociation and degradation by ECE-1 in the pathophysiology of itch. Because neuropeptide degradation in endosomes is a key mechanism in regulating $\beta$-arrestin-mediated MAPK signaling $(41,43)$, we hypothesized that ET-1 mediates activation of ERK1/2 in DRG neurons and that ECE-1 modulates the status of ET-1-triggered ERK1/2 phosphorylation. To test this hypothesis, we pretreated DRG neurons with SM-19712 $(10 \mu \mathrm{M})$ or vehicle for 60 minutes and subsequently stimulated cultured DRG neurons with ET-1 $(100 \mathrm{nM})$. Cellular lysates were analyzed by Western blotting to detect the phosphorylation status of ERK1/2 and p38. ET-1 rapidly activated ERK1/2, which peaked at 5 minutes, and ERK1/2 phosphorylation subsequently declined and was not observable 120 minutes after agonist application (Figure 4). No phosphorylation of the pain modulator p38 $(55,56)$ was induced by ET- 1 .
These data confirm a recent publication by Cevikbas et al. showing that the pruritogenic cytokine IL-31 induces phosphorylation of ERK1/2, but not p38 (57). In contrast, the time course of ET-1-induced ERK1/2 activation in DRG neurons pretreated with the ECE-1 inhibitor SM-19712 resembled that of vehicle-treated probes (Figure 4). As in DRG neurons challenged with ET-1 alone, those cotreated with ET-1 and SM-19712 demonstrated a rapid activation of ERK1/2 (within 5 minutes after agonist application). However, in marked contrast to neurons treated with ET-1 alone, we found that ECE-1 inhibition evoked a remarkably sustained ET-1-stimulated ERK1/2 activation that remained elevated over the monitored time course of 120 minutes (Figure 4).

Inbibition of ERK1/2 abolishes ET-1-induced pruritus in mice in vivo. Our in vitro data strongly indicate a potential role of ERK1/2 signaling in ET-1-induced pruritus in vivo. Consequently, we used a pharmacological approach in a mouse model of itch to determine the importance of ERK1/2 activation for ET-1-triggered pruritus in vivo. An ERK1/2 inhibitor (ERK inhibitor, $30 \mathrm{mg} / \mathrm{kg}$; Calbiochem) was administered i.p. 30 minutes prior to ET-1 application either in the nape of neck (Figure 5A) or the cheek (Figure 5B) of WT mice. This pretreatment abolished ET-1-evoked scratching behavior in both mouse models (Figure 5, A and B). Importantly, mice cotreated with ET-1 plus ERK inhibitor or with ERK inhibitor alone showed no signs of sedation, pain, or inactivity. Administration of a MEK1/ERK1/2 inhibitor (PD0325901, $10 \mathrm{mg} / \mathrm{kg}$ ) recapitulated the effect of ERK1/2 inhibitor treatment (Figure 5B). To study a more fundamental and central role of ERK1/2 in conveying itch signals, we investigated a panel of multiple pruritogens in their dependency on the MEK/ERK pathway. With the exception of histamine-induced $(100 \mu \mathrm{g} / 10 \mu \mathrm{l})$ itch, MEK/ERK inhibition suppressed the effects of all tested pruritogens (Figure $5 \mathrm{~B}$ ). Thus, in addition to ET-1, MEK1 or ERK1/2 inhibition significantly reduced or abolished the pruritogenic actions of CQ $(200 \mu \mathrm{g} / 10 \mu \mathrm{l})$ and IL-31 $(5 \mathrm{nmol} / 10 \mu \mathrm{l})$.

ETAR inbibition alleviates pruritic responses in chronic pruritic mouse models. To test whether ET-1/ETAR might be integral to the pathophysiology of pruritic diseases, we inhibited ETAR signaling in WT mice and in a chronic, oxazolone-driven pruritic mouse model (58). Local injection of BQ-123 (1 nmol/site) 30 minutes prior to ET-1 injection into the cheek (100 pmol) was sufficient to inhibit scratching behavior (Supplemental Figure 3). Chronic treatment with oxazolone for several days induced a chronic pruritic skin disorder characterized by eczema and intolerable itch. Chronification of the mouse model was established starting from day 12 and identified by chronic and stable scratching behavior. Concurrent local injection into oxazolone-applied skin with the ETAR inhibitor BQ-123 (10 nmol or $25 \mathrm{nmol} / 100 \mu \mathrm{l})$ significantly reduced the scratching behavior in chronic pruritic dermatitis (Figure 5C). We conclude that the ET-1/ETAR/ECE-1 pathway plays an important role in controlling chronic itch in mice.

ET-1 is upregulated in buman chronic pruritic diseases. In order to develop beneficial new therapies in humans, it is critical to translate findings in rodents into the human system. Although ET- 1 is a potent pruritogen in mice that acts at a picomolar range (27), the mechanism and impact of ET-1-induced pruritus in humans are poorly understood. To test whether ET-1 also accounts for itch symptoms involved in human skin diseases, we compared the immunoreactivity of ETAR and ET-1 in skin biopsies from patients with atopic dermatitis $(\mathrm{AD})(n=4)$ and prurigo nodularis $(\mathrm{PN})(n=4)$, a disease characterized by chronic, intractable 


\section{Figure 5}

ERK $1 / 2$ plays a central role in ET-1-induced itch in vivo. (A) ET-1 (10 pmol/site) was injected i.d. into the nape of neck 30 minutes after i.p. injection of an ERK1/2 inhibitor (30 mg/kg) and/or ECE-1 inhibitor SM-19712 (25 mg/kg) ( $n=9$ mice/group). (B) Scratching elicited by ET-1, IL-31, and CQ was eliminated or significantly decreased in ERK inhibitor-treated (30 mg/kg BW, targets ERK1/2) or PD0325901-treated (10 mg/kg BW, targets ERK1/2 phosphorylation) mice. Total number of scratching bouts over a 30-minute period in response to i.d. injection into the cheek of mice $(n=4)$ of a pruritic compound panel was monitored. Compound concentrations: $100 \mathrm{pmol} / 10 \mu \mathrm{l}$ ET-1, $100 \mu \mathrm{g} / 10 \mu \mathrm{l}$ histamine, $5 \mathrm{nmol} / 10 \mu \mathrm{l} \mathrm{IL}-31$, and $200 \mu \mathrm{g} / 10 \mu \mathrm{l} \mathrm{CQ}$. (C) Amelioration of itch by ETAR blockage in mice with an AD-like phenotype. Cohorts of WT mice were treated with multiple injections of oxazolone (OXA) into the nape of neck to induce chronic pruritus. Oxazolone challenge induced a robust and persistent scratching behavior in mice (baseline was evaluated on days $12-18)$. On day 18 , mice were i.d. injected into the nape of the neck with BQ-123 $(10 \mathrm{nmol} / 100 \mu \mathrm{l}$ or $25 \mathrm{nmol} / 100 \mu \mathrm{l})$. Within 30 minutes after $B Q-123$ injection, the total number of scratching bouts over a 30-minute period was determined. ${ }^{*} P<0.05$; ${ }^{* \star} P<0.01$; ${ }^{* * \star} P<0.001$. One-way ANOVA with Dunnett's post-hoc test.

pruritus, with immunoreactivity in healthy skin controls $(n=7)$. In PN (Figure 6B), immunoreactivity of ET-1 was significantly increased in all layers of the epidermis, but ETAR was not elevated, while no or very weak staining for ETAR and ET-1 was observed in healthy skin controls (Figure 6B). In AD, the amount of total ET-1 in the epidermis might be enhanced due to acanthosis, although we did not observe increased immunoreactivity on the level of a single keratinocyte (Figure 6C). Likewise, we found that ETAR immunostaining was not enhanced on keratinocytes or sensory nerves in human AD skin (not shown). We also confirmed ETAR, ET-1, and ECE-1 expression for peripheral nerves in skin biopsies taken from PN patients (Figure 6A).

ET-1 induces partially bistamine-independent pruritus in humans. To correlate the increased distribution of ET-1 and ETAR in prurigo with ET-1-induced effects in humans, we applied ET-1 to human forearm skin by iontophoresis. Corresponding to the results observed in mice (52), our data show that ET-1 elicited an itch response in human subjects that was partially independent of H1R function (Figure 6C). Preapplication of an H1R antagonist markedly, but not completely, diminished the ET-1-induced effects in human skin for the first 5 minutes after agonist application, but alleviated itch in the following monitored time interval (Figure 6C). Of note, while ET-1 induced long-lasting itch in human subjects treated with an anti-H1R antagonist, histamine failed to produce itch in this setting. The ET-1-induced local erythema was comparable to the histamine-induced erythema after iontophoresis, but no significant axon-reflex flare or wheal was observed for ET-1 (data not shown), both of which are usually observed after histamine application. This indicates that ET-1-induced pruritus in humans has a different but not completely independent mechanism from that of histamine. Thus, the combination of an ETAR antagonist with an H1R blocker may be the most efficient means of treating ET-1-induced itch in humans.

Because ET-1 has been linked to pain perception, we also asked the human subjects for non-itch responses after ET-1 application. Of note, almost all human subjects described a stinging sensation during the first 2-3 minutes after ET-1 stimulation, but a pain-related sensation was described as lasting for only
A
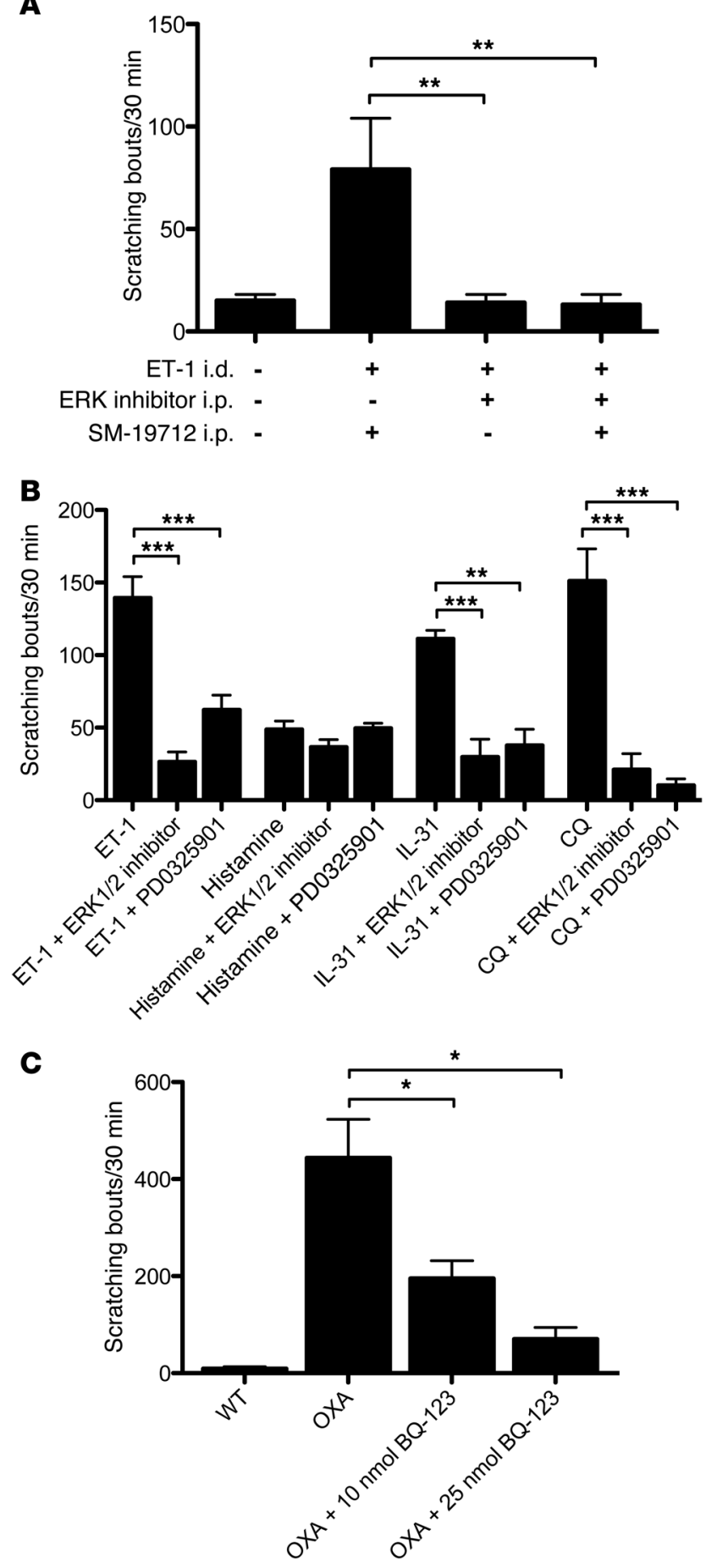

a few seconds after ET-1 application (Supplemental Figure 7). This result emphasizes the importance of translational studies to fully elucidate the importance of a mediator or receptor as a therapeutic target to treat itch or pain.

Together, our human in vivo and ex vivo findings indicate that ET-1 is increased in chronic prurigo skin diseases, is released by C fibers, keratinocytes, and endothelial cells in prurigo, and activates ETAR on unmyelinated nerve fibers. Thus, ET-1 may play an important role in human pruritus pathophysiology. 
A
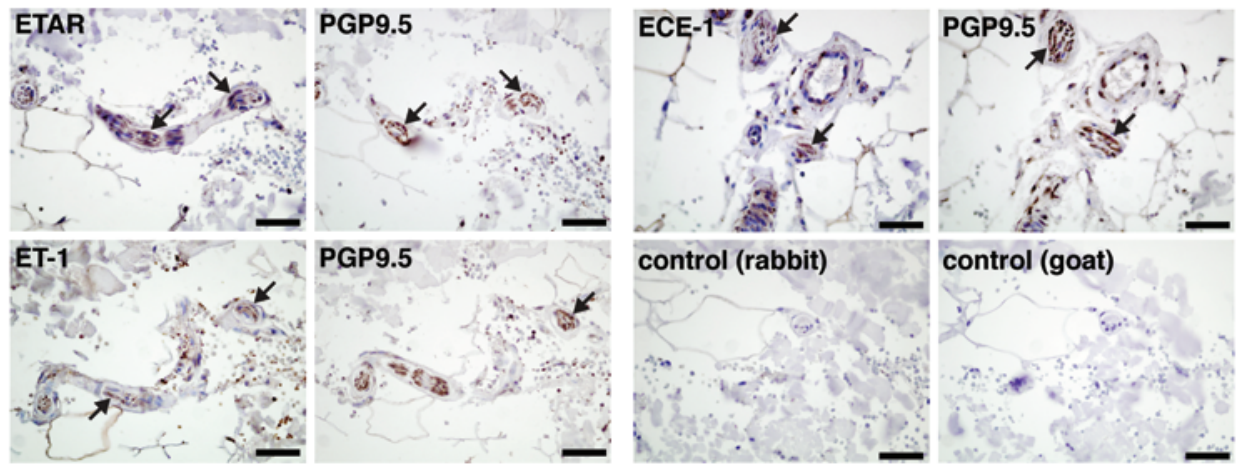

B
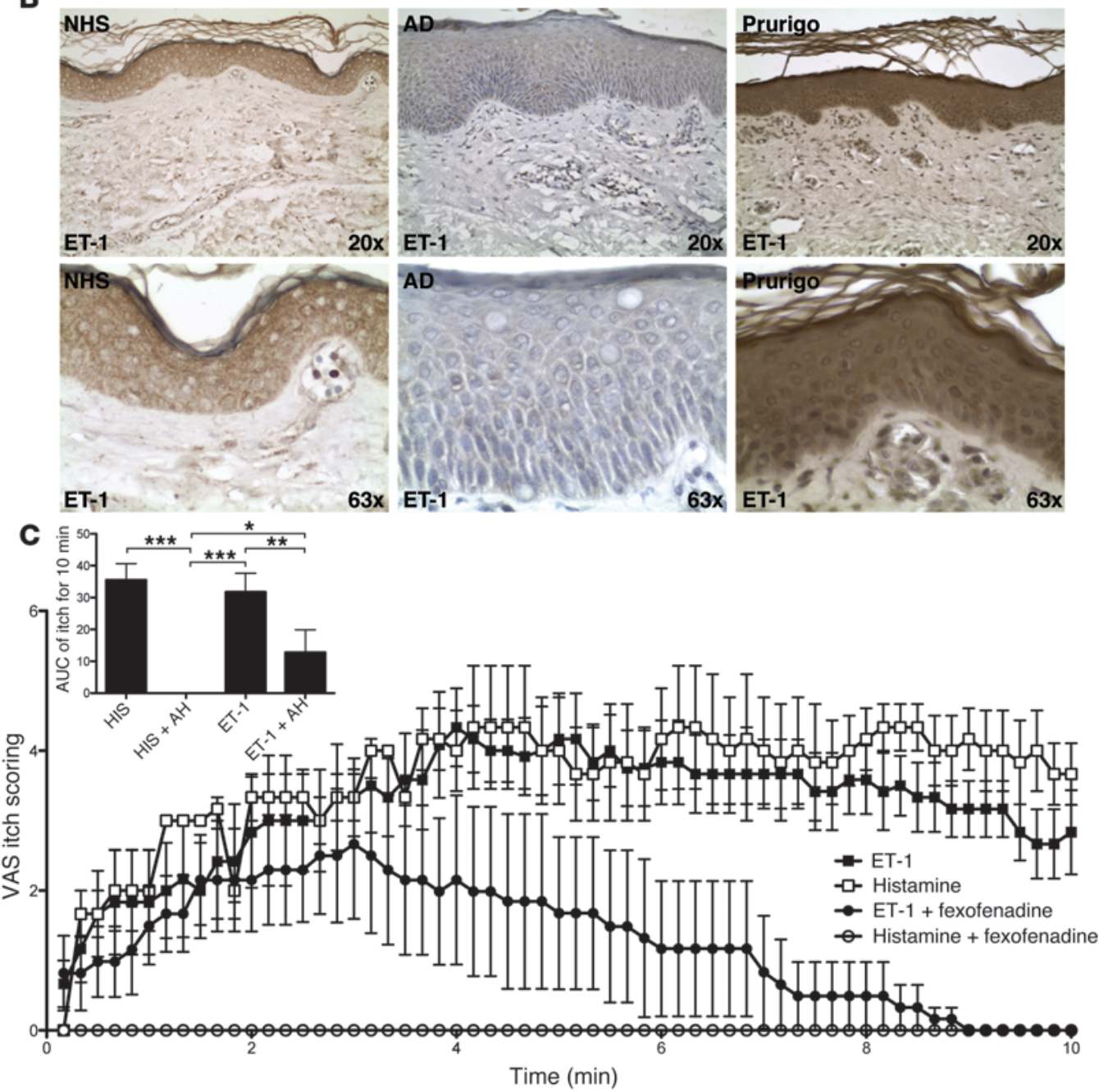

Figure 6

Increase of ET-1 and ETAR in pruritic human skin and independence of ET-1-evoked itch from H1R function in humans. (A) Localization of ET-1, ETAR, and ECE-1 in peripheral nerves of skin from patients with PN. Immunoreactivity was examined with IHC using serial sections. Positive staining for ETAR and ET-1 and (arrows) was detected for PGP9. $5^{+}$cutaneous sensory nerve fibers. Scale bars: $50 \mu \mathrm{m}$. (B) Staining for ET-1 was significantly enhanced in the epidermis of PN-affected skin compared with that of healthy skin (NHS) and AD. Samples from AD patients did not show enhanced ET-1 staining in keratinocytes, but due to acanthosis, the total harbored ET-1 may be increased when compared with healthy skin. Keratinocytes stained positively for ET-1, but fibroblasts stained negatively for ET-1. (C) Time course of itch intensity (0: no itch, 10: worst imaginable itch) for a 10-minute period after termination of a 60-second iontophoresis with histamine (white circles) and ET-1 (black circles) on the forearm skin of healthy human subjects $(n=6)$ who were orally administered a 60 -mg fexofenadine tablet (antihistamine H1R blocker) 3 hours before. ET-1 induced itch partly but not completely independently of histamine release in humans, because the antihistamine H1R blocker did not significantly block itch from 0-4 minutes, but ameliorated itch perception of volunteers from 4 minutes until the end of monitoring. Agonist-only treatment controls are shown for ET-1 (black squares) and histamine (white squares), respectively. ${ }^{\star} P<0.05$; ${ }^{* \star} P<0.01$; ${ }^{* \star} P<0.001$; error bars indicate the SEM. 


\section{Discussion}

The cellular mechanisms that regulate histamine-independent pruritus and itch-associated cell signaling in neurons are still poorly understood. Here, we describe for what we believe to be the first time that an intracellular neural peptidase, ECE-1, regulates ET-1induced pruritus and intracellular cell signaling in DRG neurons. This is based on the following evidence: ET-1, ETAR, and ECE-1 are expressed on mouse DRG neurons at the RNA and protein levels and can be colocalized on small- to medium-diameter neurons, predominantly of the peptidergic (CGRP-positive) type. ET-1, ETAR, and ECE- 1 are colocalized in the endosomes of murine DRG neurons after ETAR stimulation, suggesting that ECE-1 is important for hydrolyzing ET-1 and recycling the internalized ET-1/ETAR complex. Although we observed a colocalization of ECE-1 with the ET-1/ETAR complex in early endosomes, we hypothesized that ECE-1 hydrolyzes ET-1 in acidified endosomes (lysosomes). Our results show that ECE- 1 cleaved ET-1 at a $\mathrm{pH}$ of 5.5, but not $\mathrm{pH}$ 7.4 , indicating that ECE-1 cleaves ET-1 in lysosomes. ET-1 induced $\mathrm{Ca}^{2+}$ mobilization and ERK1/2 phosphorylation in DRG neurons. Application of ET-1 to murine skin resulted in marked scratching behavior in vivo, and ECE-1 inhibition markedly enhanced ET-1induced scratching behavior in vivo. The itch response that was induced by ET- 1 and augmented by ECE- 1 in mice in vivo was associated with a sustained ERK1/2 induction in DRG neurons, but not p38. ERK1/2 inhibition abrogated ET-1-induced scratching behavior in vivo. Finally, in humans, ET-1 induced a pruritic response independently of H1R, and its expression was upregulated in the pruritic skin of patients with chronic prurigo, suggesting a role of this pathway in human chronic pruritic disease. Based on our translational data, we show that ECE- 1 is an important regulator of ET-1-induced pruritus in mice and humans and is the first negative regulator of itch to be described on a molecular level.

Regulatory peptides released by neurons and skin cells are important mediators involved in inflammation, pain, and pruritus (59). Upon stimulation, mast cells, endothelial cells, and sensory nerves can release ET-1 (22-25). ET-1 has been described as a pain mediator $(60)$, but is also a potent itch inducer in murine and human skin $(14,27,28,33)$. ET-1 is involved in mast cell-dependent inflammation by release of mediators such as histamine and leukotriene C4 (61-64). However, histamine released from mast cells appears to be partly, but not mainly, engaged in ET-1-induced pruritus (52), indicating a direct role of neuronally expressed ETAR in pruritus.

Our study shows that ET-1-induced activation of ETAR leads to internalization of ETAR in DRG neurons and colocalization with ECE-1 in a subgroup of neurons that predominantly stain for CGRP/TRPV1 and, to a lesser extent, IB4. Our morphometric analysis demonstrates that ET-1, ETAR, and ECE-1 were coexpressed by a large proportion of small- to medium-sized DRG neurons that were also CGRP positive. Thus, ET-1 and ECE-1 may regulate the function of peptidergic neurons during pruritus, although a contribution by nonpeptidergic neurons (IB4 ${ }^{+}$) cannot be excluded. Intriguingly, the induction of ET-1-induced pruritus was not linked to a functional TRPV1 (Figure 2A and ref. 14) but rather to an operating TRPA1 channel (Figure 2A), indicating a selective itch pathway for ET-1 similar to that of MrgpA3 (18), for example, but not that of TRPV1, like histamine or serotonin $(14,65)$. However, our observation of an ET-1-induced pruritic response that was stronger in WT than in Trpa1 $1^{-/}$mice contradicts earlier results of Liang et al. (19), in which TRP inhibitors were used.
Recently, we showed that ECE-1 plays a crucial role in regulating neuropeptide receptor internalization, recycling, and cell signaling (40-43). Since ECE-1 colocalizes with ET-1 and ETAR, we hypothesized that ECE-1 regulates ET-1 function in DRG neurons. Therefore, we performed HPLC and mass spectrometric studies to determine whether ECE-1 can cleave ET-1. Indeed, ECE-1 cleaved ET-1 at the $\mathrm{Asp}^{18}-\mathrm{Ile}^{19}$ residues, resulting in two peptide fragments at an acidic $\mathrm{pH}$ of 5.5. Thus, like SP, CGRP, or somatostatin, ECE-1 internalized with the ET-1/ETAR complex into early endosomes, cleaved ET-1, and may thereby regulate the function of ET-1 in murine DRG neurons during an itch response $(27,33)$. Recently, it has been shown that ECE-1 participates in the modulation of neurogenic inflammation in mice in vivo (66). However, we believe that the results of the current study demonstrate for the first time that ECE-1 is also directly involved in ET-1-mediated pruritus and neuronal cell signaling in vivo. The fact that an ECE- 1 inhibitor augments ET-1-induced scratching behavior in mice in vivo suggests that ECE-1 regulates ET-1-induced pruritus and probably intracellular cell signaling in DRG neurons during pruritus.

The intracellular cell signaling cascades during pruritus are not well understood $(8,67)$. Therefore, we examined the itchassociated intracellular signaling pathways induced by ET-1 in DRG. We found that ET-1 induced phosphorylation of ERK1/2, but not p38. Inhibition of ECE-1 markedly prolonged ET-1induced ERK1/2 activation in DRG neurons, suggesting an antipruritic role of ECE-1 in cell signaling during ET-1-mediated pruritus. This is of importance, since endogenous antipruritic regulatory mechanisms are very poorly understood.

Very recently, Imamachi and coworkers described the existence of at least three distinct molecular pathways that contribute to the transduction of itch responses to different pruritogens (14). They report that histamine requires the presence of both PLC $\beta 3$ signaling and a functional TRPV1 channel to mediate itch information, whereas serotonin elicits itch through PLC $\beta 3$, but does not require TRPV1 actions. They also showed that ET-1-induced itch was independent of PLC $\beta 3$ function and does not require TRPV1. We demonstrate that ET-1-induced pruritus induces $\mathrm{Ca}^{2+}$ mobilization not only in WT but also in Trpv1 $1^{-/}$DRG neurons. Of note, ET-1-induced $\mathrm{Ca}^{2+}$ mobilization was slightly reduced in Trpa1-/- DRG neurons. In general, ET-1-induced scratching was not statistically different in WT mice compared with that in $\operatorname{Tr} p v 1^{-/-}, \operatorname{Tr} p v 4^{-/-}$, or $\operatorname{Tr} p m 8^{-/-}$mice. This result is in agreement with our observation that TRPA1 inhibition in WT and Trpa1 $1^{-/}$mice resulted in a reduced scratching behavior after ET-1 injection. A potential link of the ET-1 itch pathway to other functional TRPV channels such as TRPV3 is currently under investigation.

Our findings further support the concept that peptidases such as ECE- 1 contribute to the regulation of neuropeptide-mediated effects in skin diseases and pruritus $(68,69)$. Our results show that inhibition of ECE-1 leads to a sustained activation of ERK1/2. Because of its capacity to stabilize the receptor-ligand complex in relation to cell signaling, ECE-1 simultaneously contributes to receptor-mediated cell signaling as well as receptor recycling $(40-43,70)$. The impact of these two ECE-1-mediated mechanisms on human disease states is currently under investigation.

ECE-1 also regulates neurogenic inflammation and may thereby contribute to inflammatory skin diseases such as atopic dermatitis. A role of ECE-1 in the mechanism of itch, a symptom associated with many inflammatory skin diseases, has been established with this study. The observation that ET- 1 is dramatically 
enhanced in keratinocytes of prurigo, but not in those of acute $\mathrm{AD}$, is very interesting and will have to be further investigated in the future by comparing different acute and chronic pruritic diseases as well as peripheral versus central itch. In PN, a disease with chronic, often compulsive, scratching behavior, patients appear to be engaged in ET-1-induced itch that is resistant to antihistamine therapy. Given that the ETAR inhibitor showed beneficial effect in mice in vivo and that it was found to be mainly but not completely independent of histamine release from mast cells in human subjects, it will be critical to further study the impact of ETAR receptor blockers on the treatment of recalcitrant itch. Furthermore, whether ECE-1 or ERK1/2 signaling directly modulates pruritus transmission in humans and/or mice at the central nervous system level remains to be determined.

Our knowledge of whether the data from rodent studies on the molecular mechanisms of pruritus can be translated into human disease pathophysiology is very poor. Here, we provide the first evidence to our knowledge that ET-1, partially independently of histamine, induces long-lasting itch and only brief pain sensation in humans. Moreover, ET-1 is upregulated in patients with chronic itch $(\mathrm{PN})$, suggesting a role for this neural peptide and its receptor ETAR in human antihistamine-unresponsive pruritic disease, and inaugurates the peptidase ECE-1 as a regulator of ET-1-induced signaling. Of note, this study provides the first description to our knowledge of an endogenous negative regulator of itch transmission in mice and humans and may lead to new strategies to treat pruritus and various pruritic diseases.

\section{Methods}

Reagents. ET-1 and SLIGRL-NH2 were purchased from Bachem. CQ and histamine were obtained from Sigma-Aldrich. Recombinant IL-31 was a gift from S. Dillon (ZymoGenetics, Bristol-Meyers Squibb). Capsaicin and AITC were obtained from Sigma-Aldrich. The ECE-1 inhibitor SM-19712 (4-chloro-N-[(4cyano-3-methyl-1-phenil-1-hpyrazol-5-yl) amino]carbonyl] benzene sulfonamide, sodium salt) was obtained from Sigma-Aldrich. rhECE-1 was purchased from R\&D Systems. ERK1/2 inhibitor (ERK inhibitor, 328006) and MEK1/ERK1/2 inhibitor (PD0325901) were obtained from Calbiochem and Sigma-Aldrich, respectively. TRPA1 inhibitor (HC-030031) was obtained from Tocris. H1R inhibitor (diphenhydramine hydrochloride) and ETAR inhibitor (BQ-123) were obtained from Sigma-Aldrich.

Antibodies. Anti-ECE-1 and anti-EEA1 antibodies were purchased from Santa Cruz Biotechnology, Inc. Anti-ET-1 and anti-CGRP antibodies were purchased from Bachem. Anti-ETAR, anti-ETBR, anti-PGP9.5 rabbit polyclonal antibody, and anti- $\beta$-actin antibody were obtained from Abcam. Biotinylated IB4 was purchased from Vector Laboratories. FITCconjugated anti-goat or rabbit IgG and Dylight 488-conjugated anti-biotin or anti-guinea pig IgG were obtained from Jackson ImmunoResearch. Alexa Fluor 568-conjugated anti-rabbit or goat IgG was purchased from Invitrogen. Anti-pERK1/2, anti-ERK1/2, anti-pp38, and anti-p38 were obtained from Cell Signaling Technology. Peroxidase-labeled anti-rabbit or anti-goat secondary antibodies were purchased from GE Healthcare.

Mice. Adult C57bl/6 mice (Harlan Laboratories) were maintained under specific pathogen-free conditions with free access to food and water. $\operatorname{Tr} p v 1^{-/-}, \operatorname{Tr} p v 4^{-/}$, and $\operatorname{Tr} p a 1^{-/-}$mice were gifts from David Julius (Department of Physiology, UCSF). Trpm $8^{-/-}$mice were obtained from The Jackson Laboratory.

Isolation of DRG neurons and dorsal skin. Mice were deeply anesthetized by i.p. injection of a mixture of $0.2 \%$ xylazine, $1 \%$ ketamine, and $0.9 \% \mathrm{NaCl}$ (Ceva Sante Animale) and then perfused transcardially with $\mathrm{Ca}^{2+}$-free and
$\mathrm{Mg}^{2+}$-free PBS. When samples were dedicated to immunohistochemical (IHC) or immunofluorescence analysis, transcardial perfusion was performed using PBS containing 4\% PFA.

Primary DRG culture. Harvested DRG neurons were incubated with Hank's balanced salt solution (BSS) containing $1.3 \mathrm{mg} / \mathrm{ml}$ papain (Sigma-Aldrich) and $0.65 \mathrm{mg} / \mathrm{ml} \mathrm{L-cysteine} \mathrm{(Sigma-Aldrich)} \mathrm{for} 10$ minutes at $37^{\circ} \mathrm{C}$ and then incubated with Hank's BSS containing $3 \mathrm{mg} / \mathrm{ml}$ collagenase (Sigma-Aldrich) for 10 minutes at $37^{\circ} \mathrm{C}$. Digests were washed with complete medium consisting of DMEM with Earle's BSS medium supplemented with $10 \%(\mathrm{v} / \mathrm{v})$ horse serum (Sigma-Aldrich), $2 \mathrm{mM}$ L-glutamine, $100 \mathrm{U} / \mathrm{ml}$ penicillin, $100 \mu \mathrm{g} / \mathrm{ml}$ streptomycin (all from PAA Laboratories), $1 \times$ GIBCO MEM Vitamin Solution (Invitrogen), and 1× N1 medium supplement (Sigma-Aldrich). The cell strain suspension was filtered through a $100-\mu \mathrm{m}$ cell strainer and cultured in dishes with or without poly-D-lysine (Sigma-Aldrich) and laminin-coated glass coverslips (Sigma-Aldrich) containing complete media overnight. Cells were preincubated with vehicle or SM-19712 (10 $\mu \mathrm{M}) 2$ hours before agonist stimulation. Inhibitor incubation was performed throughout the experiments. Cells were stimulated with vehicle or ET-1 (100 nM). For calcium imaging, upper- to mid-cervical mouse DRGs were enzymatically digested and processed.

$R T-P C R$. Total RNA was extracted from homogenized samples of DRG neurons or skin using TRIzol reagent (Invitrogen) according to the manufacturer's instructions. To avoid DNA contamination, total RNA was treated with $10 \mathrm{U}$ of RQ1 RNase-Free DNase (Promega). A quantity of $1 \mu \mathrm{g}$ of whole RNA was reverse transcribed with the RevertAid First Strand cDNA Synthesis Kit (Fermentas). Subsequent RT-PCRs were carried out using GoTaq DNA polymerase from Promega. The primers for mouse ET-1 (235 bp), ECE-1(54 bp), ETAR (129 bp), ETBR (240 bp), and $\beta$-actin (147 bp) were used as previously reported (71). The primer sequences were as follows: ET-1 (mouse, NM_010104.3) forward: 5'-GGAAACTACGAAGGTTGGAGGC-3', reverse: 5'-CTGTAGAAGCCACACAGATGGTCT-3'; ECE-1 (mouse, NM_199307.2) forward: 5'-GTGGCATTGGTGTCGTAGTG-3', reverse: 5'-CTTGATCATCGAAAGCGTGA-3'; ETAR (mouse, NM_010332.2) forward: 5'-GCTGGTTCCCTCTTCACTTAAGC- $3^{\prime}$, reverse: $5^{\prime}$-TCATGGTTGCCAGGTTAATGC- $3^{\prime}$; and $\beta$-actin (mouse, NM_007392.3) forward: 5'-TTGCTGACAGGATGCAGAAG-3', reverse: 5'-TGATCCACATCTGCTGGAAG-3'.

PCR conditions involved an annealing temperature of $60^{\circ} \mathrm{C}$ for 35 cycles. PCR products were separated with a $2 \%(\mathrm{w} / \mathrm{v})$ agarose gel.

SDS-PAGE and Western blotting. Lysates of homogenized samples of DRG neurons, skin, or whole-cell lysates were homogenized in protein lysis buffer containing a protease inhibitor mixture (Roche Applied Science) and sonicated; cell debris was removed by centrifugation $\left(14,000 \mathrm{~g}\right.$ at $4{ }^{\circ} \mathrm{C}$ for 10 minutes). Samples were boiled in sample buffer (50 mM Tris-HCL, $\mathrm{pH}$ $6.8 ; 2 \%[\mathrm{w} / \mathrm{v}] \mathrm{SDS} ; 0.1 \% \mathrm{w} / \mathrm{v}$ bromophenol blue; $10 \%$ [v/v] glycerol; and $2.5 \%$ $[\mathrm{v} / \mathrm{v}] 2$-mercaptoethanol) for 5 minutes, separated by SDS-PAGE (7\% or $10 \%$ acrylamide), and blotted onto a nitrocellulose membrane (Amersham Biosciences). Membrane blocking was performed with milk powder (5\% w/v; Sigma-Aldrich) in Tris-buffered saline with $0.05 \%(\mathrm{v} / \mathrm{v})$ Tween 20 (Sigma-Aldrich) for 1 hour. Primary antibodies were applied overnight at $4^{\circ} \mathrm{C}$. After five washes with PBS-Tween 20, the membrane was incubated with a secondary antibody for 2 hours at room temperature. Immunoreactive polypeptides were visualized using the ECL Plus Western Blotting Detection Kit (GE Healthcare).

Immunofluorescence of tissues. Tissue samples (10- $\mu \mathrm{m}$ sections of DRG neurons) were postfixed in a $4 \%$ PFA solution for 2 hours at $4{ }^{\circ} \mathrm{C}$, transferred to a $30 \%$ sucrose solution, embedded in Neg-50 (Thermo Fisher Scientific) for 24 hours at $4^{\circ} \mathrm{C}$, and stored at $-80^{\circ} \mathrm{C}$ until use. Sections were washed in PBS and incubated with $2 \%(\mathrm{w} / \mathrm{v})$ BSA, $5 \%(\mathrm{v} / \mathrm{v})$ horse serum, and $0.3 \%$ (v/v) Triton X-100 in PBS for 1 hour. Sections were incubated with primary 
antibodies overnight at $4{ }^{\circ} \mathrm{C}$, then washed with PBS and incubated with secondary antibodies at room temperature for 2 hours (72).

Confocal microscopy. Confocal images were acquired using a Zeiss LSM510 META confocal microscope (Zeiss). Tissue staining images were taken with a $\times 40, \times 20$, or $\times 10$ magnification objective in the inverted configuration. Confocal images for primary cultured DRG cells were obtained with a $\times 63$ magnification objective in the inverted configuration. For all confocal images, a regular-phase transmission image was obtained. In DRGs, the subcellular distribution of ETAR, ECE-1, and ET-1 were analyzed from captured images using Image J software (NIH) as described previously $(73,74)$. To analyze the subcellular distribution of ETAR, plasma membrane fluorescence was calculated by subtracting cytoplasmic pixel counts from total cell pixel counts. To analyze the fluorescence intensity of ET-1 and ECE-1 over time, total cell pixel counts were calculated.

IHC. Six-micrometer paraffin sections were deparaffinized, rehydrated, and heated in a steamer (MultiGourmet Plus FS20; Braun) for 25 minutes in Target Retrieval Solution (Dako). Sections were allowed to cool down in Target Retrieval Solution for 20 minutes. Endogenous peroxidase activity was quenched with $100 \mathrm{mM} \mathrm{NaN} 30.1 \%$ (w/v) $\mathrm{H}_{2} \mathrm{O}_{2}$ in PBS for 20 minutes at room temperature. After washing with PBS, sections were blocked with $2 \%(\mathrm{w} / \mathrm{v}) \mathrm{BSA}, 5 \%(\mathrm{w} / \mathrm{v})$ horse serum, and $0.3 \%(\mathrm{v} / \mathrm{v})$ Triton-X 100 (USB Corporation) for 1 hour at room temperature. Sections were incubated with primary antibodies overnight at $4{ }^{\circ} \mathrm{C}$ in a humid chamber. After being rinsed with PBS, sections were incubated with secondary antibodies for 1 hour at room temperature (EnVision+ System HRP-labeled polymer, anti-rabbit, anti-goat, or anti-sheep; Dako). Immunoreactivity was detected using the Liquid DAB+ Substrate Chromogen System (Dako). Nuclei were counterstained with Hematoxylin QS (Vector Laboratories) and mounted with Aquamount (BDH). Stained sections were examined using the Axiophot microscope (Zeiss) equipped with a DP70 CCD camera (Olympus). Morphometric analysis of images was performed using DP-Soft 3.2 software (Olympus).

Scratching behavior. Mice were placed individually in a glass cage $(13 \times 13 \times 20 \mathrm{~cm})$ for at least 30 minutes before behavioral studies. ET-1 ( $1 \mathrm{pmol}-1 \mathrm{nmol} /$ site in $0.9 \% \mathrm{NaCl})$ or vehicle was administered i.d. into the nape of the neck or cheek of the mouse. Scratching and wiping behavior was videotaped for 30 minutes with personnel kept out of the observation room and was analyzed in a double-blinded fashion. Analysis of the video footage to determine the number of scratching bouts included only scratching with the hind paw toward the injected site and wiping of the right cheek with the front paw. A series of scratching movements within 1 second was counted as 1 bout of scratching. SM-19712 (25 mg/kg BW in 0.9\% NaCl), ERK1/2 inhibitor $(30 \mathrm{mg} / \mathrm{kg}$ $\mathrm{BW}$ in $0.9 \% \mathrm{NaCl}, 10 \% \mathrm{DMSO}), \mathrm{HC}-030031(30 \mathrm{mg} / \mathrm{kg} \mathrm{BW}$ in $0.9 \%$ $\mathrm{NaCl}$ ), $\mathrm{PD} 0325901$ (in $0.9 \% \mathrm{NaCl}, 10 \% \mathrm{DMSO}$ ), or vehicle was injected i.p. 30 minutes before ET-1 injection. The H1R inhibitor diphenhydramine hydrochloride $\left(3 \mathrm{mg} / \mathrm{kg}\right.$ in $\mathrm{H}_{2} \mathrm{O}$ ) was applied orally 30 minutes before ET-1 injection. BQ-123 $(1-25 \mathrm{nmol}$ in $0.9 \% \mathrm{NaCl})$ was injected i.d. 30 minutes before ET-1 injection.

Chronic contact dermatitis. Mice were sensitized by topical treatment with $5 \%$ oxazolone $(10 \mu \mathrm{l})$, which was applied to the left ear. After 7 days, oxazolone-sensitized mice were topically treated with $0.2 \%$ oxazolone $(60 \mu \mathrm{l})$ on the flank every other day for a total of 2 weeks. Skin biopsies of mice were obtained on the last day of oxazolone treatment.

Chronic pruritus model. WT mice were treated one time with $10 \%$ oxazolone in acetone/olive oil $(4: 1 \mathrm{v} / \mathrm{v})$ on the shaved nape of the neck $(100 \mu \mathrm{l})$. After a resting period of 7 days, mice were treated with $1 \%$ oxazolone in acetone/ olive oil $(4: 1 \mathrm{v} / \mathrm{v})$ on the nape of the neck $(100 \mu \mathrm{l})$ every other day for an additional 10 days. Baseline scratching behavior was evaluated for $30 \mathrm{~min}$ utes on days $0,12,14,16$, and 18 .
Immunofluorescence of cultured cells. Cultured and stimulated DRG cells were fixed in 4\% PFA and $100 \mathrm{mM}$ PBS ( $\mathrm{pH} 7.4$ ), washed and incubated in PBS containing $1 \%$ FCS and $0.1 \%$ saponin for 30 minutes, and incubated with primary antibodies overnight at $4{ }^{\circ} \mathrm{C}$. Cells were washed, incubated with secondary antibodies for 2 hours at room temperature, then washed and mounted.

Peptide degradation by ECE-1. ET-1 $(250 \mu \mathrm{M})$ was incubated with rhECE-1 $(100 \mathrm{nM})$ in $50 \mathrm{mM} \mathrm{Mes} / \mathrm{KOH}(\mathrm{pH} 5.5)$ or $50 \mathrm{mM}$ Tris- $\mathrm{HCl}$ ( $\mathrm{pH} 7.4)$ for 0 to 90 minutes at $37^{\circ} \mathrm{C}$. Reactions were stopped by adding trifluoroacetic acid (TFA). Samples were run on a reverse-phase HPLC and analyzed using an ABI 4700 MALDI TOF/TOF mass spectrometer. The predicted masses of the peptides were calculated using the MS-Product feature of the Protein Prospector program (http://prospector.ucsf.edu/prospector/mshome.htm).

Measurement of single-cell $\left[\mathrm{Ca}^{2+}\right]_{i}$. DRG cells were seeded on glass coverslips (Bellco Coverslips, $18 \mathrm{~mm}$; Electron Microscopy Sciences) coated with poly-D-lysine and laminin overnight. Two hours before experiments, neurons were washed and kept in a medium containing $1 \%$ horse serum. Coverslips were washed with HEPES buffer and subsequently incubated with complete medium containing $2.5 \mu \mathrm{M}$ Fluo-3 AM (Invitrogen) for 30 minutes at $37^{\circ} \mathrm{C}$. Medium was removed, and extracellular contamination with Fluo-3 AM was eliminated by rinsing with HEPES buffer. Glass coverslips were transferred to a perfusion chamber, and the cells were perfused with medium for 10 minutes to allow complete de-esterification of cytosolic dye. ET-1 at a concentration of $100 \mathrm{nM}$ was then administered. Fluorescence signals were measured with a confocal laser scanning microscope (Zeiss LSM710) using an excitation wavelength of $488 \mathrm{~nm}$. Emission signals between $505 \mathrm{~nm}$ and 530 were recorded. Two-dimensional images were recorded every 0.78 seconds. Relative $\left[\mathrm{Ca}_{2}^{+}\right]_{\mathrm{i}}$ levels are shown as pseudo-ratios $\left(\mathrm{F} / \mathrm{F}_{0}\right)$.

Calcium imaging. Upper- to mid-cervical DRGs removed from mice were enzymatically digested as mentioned above. Cells were plated on poly-D-lysine-coated glass coverslips and cultured for 16 to 24 hours. Cells were incubated in Ringer's solution ( $\mathrm{pH} 7.4 ; 140 \mathrm{mM} \mathrm{NaCl}, 4 \mathrm{mM}$ $\mathrm{KCl}, 2 \mathrm{mM} \mathrm{CaCl} 2,1 \mathrm{mM} \mathrm{MgCl} 2,10 \mathrm{mM}$ HEPES, and $4.54 \mathrm{mM} \mathrm{NaOH}$ ) with $10 \mu \mathrm{M}$ of Fura-2 AM and $0.05 \%$ of Pluronic F-127 (Invitrogen). Coverslips were mounted on a custom-made aluminum perfusion block and viewed through an inverted fluorescence microscope (Nikon Eclipse TS100). Fluorescence was excited by UV light at alternating wavelengths of $340 \mathrm{~nm}$ and $380 \mathrm{~nm}$, and the emitted light was collected via a CoolSnap camera attached to a Lambda LS lamp and a Lambda optical filter changer (Sutter Instrument Company). Ratiometric measurements were made every 3 seconds using Simple PCI software (Compix, Inc.). Approximately 40 cells were observed per dish and subjected to the identical stimulus sequence. Solutions were delivered by a solenoid-controlled 8-channel perfusion system (ValveLink; AutoMate Scientific) at a flow rate of $6 \mathrm{ml} /$ minute. ET-1 $(1 \mu \mathrm{M})$ and histamine $(100 \mu \mathrm{M})$, SLIGRL-NH2 $(100 \mu \mathrm{M})$, or CQ $(300 \mu \mathrm{M})$ were delivered, usually in this order. After applications of pruritogens, $1 \mu \mathrm{M}$ capsaicin or $100 \mu \mathrm{M}$ allyl isothiocyanate (AITC) and $144 \mathrm{mM}$ potassium were applied in this order. The stimulus duration was 30 seconds ( 10 seconds for capsaicin and AITC). Ratios were normalized to the prestimulus baseline. Cells were judged to be responsive if the ratio value increased by more than $10 \%$ of the resting level following chemical application.

Iontophoresis in buman subjects. Six healthy adult human subjects ( 4 women and 2 men, mean age 30.2 years) participated in the experiment after giving informed consent. Three hours after a 60-mg tablet of fexofenadine, a commercially available antihistamine H1R blocker, was administered orally to the subjects, histamine dehydrochloride $(10 \mathrm{mg} / \mathrm{ml}$ in distilled water; Sigma-Aldrich) and ET-1 $(10 \mu \mathrm{g} / \mathrm{ml}$ in distilled water; Sigma-Aldrich) were iontophoretically applied to the volar side of the left 
forearm, as has been described previously (75). Histamine or ET-1 was applied in random order at 10-minute intervals, and both the subjects and the examiner were blinded to the treatment type. For iontophoresis of each substance, a constant current of $0.2 \mathrm{~mA}$ was applied to the skin for 60 seconds through the iontophoresis system, and round-shaped counter electrodes of $5-\mathrm{mm}$ and $15-\mathrm{mm}$ diameter, respectively, were placed $5 \mathrm{~cm}$ apart. The subjects were asked to describe the itch intensity on a 0 (no itch) to 10 (worst itch imaginable) scale every 10 seconds for 10 minutes after the termination of iontophoresis.

Statistics. Data are presented as the mean \pm SEM. Analyses were performed using GraphPad Prism 4.0 software (GraphPad Software). Between-group comparisons were performed by a 2-tailed Student's $t$ test. Multiple comparisons within groups were performed by repeated-measures 1-way ANOVA, followed by a Bonferroni's post-hoc test or Dunnett's post-hoc test unless otherwise indicated. Statistical significance was set at $P<0.05$.

Study approval and human subjects. Written and verbal informed consent was obtained from participants prior to enrollment in the study, in accordance with Declaration of Helsinki principles. Approval of the human studies was granted by the ethics committee of the University of Münster. Patients received no topical medications 2 weeks before undergoing the biopsy procedures. The skin samples included 4 lesional ADs (from 2 males and 2 females, $20-60$ years of age, mean age 30.8 years), 4 PNs (from 2 males and 2 females, 25-82 years of age, mean age 47.4 years), and 7 normal skin samples obtained from postoperative samples (from 4 males and 3 females, $20-86$ years of age, mean age 45.6 years). Iontophoresis is a noninvasive in vivo method used in humans. The study was performed in volunteers ( 2 males and 4 females, 25-40 years of age, mean age 30.2 years). Results were analyzed in a blinded fashion to guarantee the independence of the results. None of the participants had skin lesions or a history of AD including allergic rhinitis or asthma.

Study approval for mouse experiments. All animal experiments were approved by the IACUC of UCSF and were conducted in accordance with NIH guidelines for the care and use of laboratory animals and with those of the Office for Environment, Nature and Municipal Affairs of North
Rhine-Westphalia (Landesamt für Natur, Umwelt und Verbraucherschutz Nordrhein-Westfalen, Germany).

\section{Acknowledgments}

The technical assistance of Heike Hinte, Diana Below, Ronald Manlapaz, Andrea Poppe, and Wendy Cedron is gratefully acknowledged. We thank Fabian Bandrowski, Randal Maile, and Ingo H. Engels for critically reading the manuscript. This work was supported by grants from the following organizations: NIH/ NIAMS (AR059402-01); Deutsche Forschungsgemeinschaft (DFG) (STE1014/2-2, to M. Steinhoff); Centre de Recherches d'Investigations Epidermiques et Sensorielles (CERIES), Paris, France (to M. Steinhoff); Toray Science Foundation, Japan (to M. Steinhoff); Leo Foundation, Denmark (to M. Steinhoff); DFG (CE165/1-1, to F. Cevikbas); DFG (KE1672/1-1, to C. Kempkes); Innovative Medizinische Forschung (IMF) (BU111220, to J. Buddenkotte); Japan Society for the Promotion of Science (JSPS) KAKENHI (25860424, to M. Kido-Nakahara); National Health and Medical Research Council (NHMRC) (63303 and 103188, to N.W. Bunnett); Monash University (to N.W. Bunnett); and NIH (DK080787, to A. Bhargava). Mass spectrometric analysis was provided by the Bio-Organic Biomedical Mass Spectrometry Resource of UCSF (A.L. Burlingame, Director), which is supported by the Biomedical Technology Research Centers program of the NIH National Institute of General Medical Sciences (NIH NIGMS 8P41GM103481).

Received for publication October 12, 2012, and accepted in revised form February 13, 2014.

Address correspondence to: Martin Steinhoff, Department of Dermatology and Charles Institute for Translational Dermatology, University College Dublin, Belfield, Dublin-4, Ireland. Phone: 353.1.716.6261; Fax: 353.1.716.6265; E-mail: Martin.Steinhoff@ucd.ie.
1. Steinhoff M, Cevikbas F, Yeh I, Chong K, Buddenkotte J, Ikoma A. Evaluation and management of a patient with chronic pruritus. J Allergy Clin Immunol. 2012;130(4):1015-6 e7.

2. Rothman S. Physiology of itching. Physiol Rev. 1941;21(2):357-3810031-93331522-1210.

3. Paus R, Schmelz M, Biro T, Steinhoff M. Frontiers in pruritus research: scratching the brain for more effective itch therapy. J Clin Invest. 2006; 116(5):1174-1186.

4. Ikoma A, Steinhoff M, Stander S, Yosipovitch G, Schmelz M. The neurobiology of itch. Nat Rev Neurosci. 2006;7(7):535-547.

5. Steinhoff M, Bienenstock J, Schmelz M, Maurer M, Wei E, Biro T. Neurophysiological, neuroimmunological, and neuroendocrine basis of pruritus. J Invest Dermatol. 2006;126(8):1705-1718.

6. Schmelz M, Schmidt R, Bickel A, Handwerker $\mathrm{HO}$, Torebjork HE. Specific C-receptors for itch in human skin. J Neurosci. 1997;17(20):8003-8008.

7. Schmelz M, Schmidt R, Weidner C, Hilliges M, Torebjork HE, Handwerker HO. Chemical response pattern of different classes of C-nociceptors to pruritogens and algogens. J Neurophysiol. 2003; 89(5):2441-2448.

8. Buddenkotte J, Steinhoff M. Pathophysiology and therapy of pruritus in allergic and atopic diseases. Allergy. 2010;65(7):805-821.

9. Andrew D, Craig AD. Spinothalamic lamina I neurons selectively sensitive to histamine: a central neural pathway for itch. Nat Neurosci. 2001;4(1):72-77.

10. Carstens E. Responses of rat spinal dorsal horn neurons to intracutaneous microinjection of hista- mine, capsaicin, and other irritants. J Neurophysiol. 1997;77(5):2499-2514.

11. Handwerker HO, Forster C, Kirchhoff C. Discharge patterns of human C-fibers induced by itching and burning stimuli. J Neurophysiol. 1991;66(1):307-315.

12. Drzezga A, et al. Central activation by histamine-induced itch: analogies to pain processing: a correlational analysis of $\mathrm{O}-15 \mathrm{H} 2 \mathrm{O}$ positron emission tomography studies. Pain. 2001;92(1-2):295-305.

13. Mochizuki H, Tashiro M, Kano M, Sakurada Y, Itoh M, Yanai K. Imaging of central itch modulation in the human brain using positron emission tomography. Pain. 2003;105(1-2):339-346.

14. Imamachi N, et al. TRPV1-expressing primary afferents generate behavioral responses to pruritogens via multiple mechanisms. Proc Natl Acad Sci U S A. 2009;106(27):11330-11335.

15. Ross SE. Pain and itch: insights into the neural circuits of aversive somatosensation in health and disease. Curr Opin Neurobiol. 2011;21(6):880-887.

16. Akiyama T, Carstens E. Neural processing of itch. Neuroscience. 2013;250:697-714.

17. Han SK, Mancino V, Simon MI. Phospholipase Cbeta 3 mediates the scratching response activated by the histamine $\mathrm{H} 1$ receptor on C-fiber nociceptive neurons. Neuron. 2006;52(4):691-703.

18. Wilson SR, et al. TRPA1 is required for histamine-independent, Mas-related G protein-coupled receptor-mediated itch. Nat Neurosci. 2011;14(5):595-602.

19. Liang J, Ji Q, Ji W. Role of transient receptor potential ankyrin subfamily member 1 in pruritus induced by endothelin-1. Neurosci Lett. 2011;492(3):175-178.
20. Guruli G, Pflug BR, Pecher S, Makarenkova $V$, Shurin MR, Nelson JB. Function and survival of dendritic cells depend on endothelin-1 and endothelin receptor autocrine loops. Blood. 2004;104(7):2107-2115.

21. Spirig R, Potapova I, Shaw-Boden J, Tsui J, Rieben R, Shaw SG. TLR2 and TLR4 agonists induce production of the vasoactive peptide endothelin-1 by human dendritic cells. Mol Immunol. 2009;46(15):3178-3182.

22. Yanagisawa $\mathrm{M}$, et al. A novel potent vasoconstrictor peptide produced by vascular endothelial cells. Nature. 1988;332(6163):411-415.

23. Giaid A, et al. Endothelin 1, an endothelium-derived peptide, is expressed in neurons of the human spinal cord and dorsal root ganglia. Proc Natl Acad Sci US A. 1989;86(19):7634-7638.

24. Ehrenreich $\mathrm{H}$, et al. Endothelins belong to the assortment of mast cell-derived and mast cellbound cytokines. New Biol. 1992;4(2):147-156.

25. Maurer M, et al. Mast cells promote homeostasis by limiting endothelin-1-induced toxicity. Nature. 2004;432(7016):512-516.

26. MacCumber MW, Ross CA, Snyder SH. Endothelin in brain: receptors, mitogenesis, and biosynthesis in glial cells. Proc Natl Acad Sci U S A. 1990;87(6):2359-2363.

27. Trentin PG, Fernandes MB, D’Orleans-Juste P, Rae GA. Endothelin-1 causes pruritus in mice. Exp Biol Med (Maywood). 2006;231(6):1146-1151.

28. Katugampola R, Church MK, Clough GF. The neurogenic vasodilator response to endothelin-1: a study in human skin in vivo. Exp Physiol. 2000;85(6):839-846. 
29. Davar G, Hans G, Fareed MU, Sinnott C, Strichartz $\mathrm{G}$. Behavioral signs of acute pain produced by application of endothelin-1 to rat sciatic nerve. Neuroreport. 1998;9(10):2279-2283.

30. Gokin AP, Fareed MU, Pan HL, Hans G, Strichartz GR, Davar G. Local injection of endothelin-1 produces pain-like behavior and excitation of nociceptors in rats. J Neurosci. 2001;21(14):5358-5366.

31. Raffa RB, Schupsky JJ, Lee DK, Jacoby HI. Characterization of endothelin-induced nociception in mice: evidence for a mechanistically distinct analgesic model. J Pharmacol Exp Ther. 1996;278(1):1-7.

32. Raffa RB, Schupsky JJ, Jacoby HI. Endothelin-induced nociception in mice: mediation by ETA and ETB receptors. J Pharmacol Exp Ther. 1996; 276(2):647-651.

33. McQueen DS, Noble MA, Bond SM. Endothelin-1 activates ETA receptors to cause reflex scratching in BALB/c mice. Br J Pharmacol. 2007;151(2):278-284.

34. Ferreira SH, Romitelli M, de Nucci G. Endothelin-1 participation in overt and inflammatory pain. J Cardiovasc Pharmacol. 1989;13(suppl 5):S220-S222.

35. Wenzel RR, Zbinden S, Noll G, Meier B, Luscher TF. Endothelin-1 induces vasodilation in human skin by nociceptor fibres and release of nitric oxide. Br J Clin Pharmacol. 1998;45(5):441-446.

36. Dahlof B, Gustafsson D, Hedner T, Jern S, Hansson $\mathrm{L}$. Regional haemodynamic effects of endothelin-1 in rat and man: unexpected adverse reaction. J Hypertens. 1990;8(9):811-817.

37. Kedzierski RM, Yanagisawa M. Endothelin system: the double-edged sword in health and disease. Annu Rev Pharmacol Toxicol. 2001;41:851-876.

38. Plant TD, et al. Endothelin potentiates TRPV1 via ETA receptor-mediated activation of protein kinase C. Mol Pain. 2007;3:35

39. Bremnes T, Paasche JD, Mehlum A, Sandberg C, Bremnes B, Attramadal H. Regulation and intracellular trafficking pathways of the endothelin receptors. J Biol Chem. 2000;275(23):17596-17604.

40. Roosterman D, et al. Endothelin-converting enzyme 1 degrades neuropeptides in endosomes to control receptor recycling. Proc Natl Acad Sci U S A. 2007;104(28):11838-11843

41. Padilla BE, et al. Endothelin-converting enzyme-1 regulates endosomal sorting of calcitonin receptor-like receptor and $\beta$-arrestins. J Cell Biol. 2007; 179(5):981-997.

42. Roosterman D, et al. Endothelin-converting enzyme-1 degrades internalized somatostatin-14. Endocrinology. 2008;149(5):2200-2207.

43. Cottrell GS, et al. Endosomal endothelin-converting enzyme-1: a regulator of $\beta$-arrestin-dependent ERK signaling. J Biol Chem. 2009;284(33):22411-22425.

44. Xu D, et al. ECE-1: a membrane-bound metalloprotease that catalyzes the proteolytic activation of big endothelin-1. Cell. 1994;78(3):473-485.

45. Shim WS, et al. TRPV1 mediates histamine-induced itching via the activation of phospholipase A2 and 12-lipoxygenase. J Neurosci. 2007;27(9):2331-2337.

46. Costa R, et al. Evidence for the role of neurogenic inflammation components in trypsin-elicited scratching behaviour in mice. $\mathrm{Br} J$ Pharmacol. 2008;154(5):1094-1103

47. Gouadon E, et al. Endothelin evokes distinct calcium transients in neuronal and non-neuronal cells of rat olfactory mucosa primary cultures. Neuroscience. 2010;165(2):584-600.

48. Yohn JJ, et al. Autoregulation of endothelin-1 secretion by cultured human keratinocytes via the endothelin B receptor. Biochim Biophys Acta. 1994;1224(3):454-458.

49. Liu Q, et al. Sensory neuron-specific GPCR Mrgprs are itch receptors mediating chloroquine-induced pruritus. Cell. 2009;139(7):1353-1365.

50. Wilson SR, et al. The epithelial cell-derived atopic dermatitis cytokine TSLP activates neurons to induce itch. Cell. 2013;155(2):285-295.

51. Gomes LO, Hara DB, Rae GA. Endothelin-1 induces itch and pain in the mouse cheek model. Life sciences. 2012;91(13-14):628-633.

52. Liang J, Kawamata T, Ji W. Molecular signaling of pruritus induced by endothelin-1 in mice. Exp Biol Med (Maywood). 2010;235(11):1300-1305.

53. Shimada SG, LaMotte RH. Behavioral differentiation between itch and pain in mouse. Pain. 2008;139(3):681-687.

54. Wang J, Chiou WJ, Gagne GD, Wu-Wong JR. Internalization of type-A endothelin receptor.J Cardiovasc Pharmacol. 2000;36(5 suppl 1):S61-S65.

55. Murphy JE, Padilla BE, Hasdemir B, Cottrell GS, Bunnett NW. Endosomes: a legitimate platform for the signaling train. Proc Natl Acad Sci U S A. 2009;106(42):17615-17622.

56. Schafers M, Svensson CI, Sommer C, Sorkin LS Tumor necrosis factor- $\alpha$ induces mechanical allodynia after spinal nerve ligation by activation of p38 MAPK in primary sensory neurons. J Neurosci. 2003;23(7):2517-2521.

57. Cevikbas F, et al. A sensory neuron-expressed IL-31 receptor mediates $\mathrm{T}$ helper cell-dependent itch: Involvement of TRPV1 and TRPA1. J Allergy Clin Immunol. 2014;133(2):448-460.

58. Man MQ, et al. Characterization of a hapten-induced, murine model with multiple features of atopic dermatitis: structural, immunologic, and biochemical changes following single versus multiple oxazolone challenges. J Invest Dermatol. 2008;128(1):79-86.

59. Roosterman D, Goerge T, Schneider SW, Bunnett NW, Steinhoff M. Neuronal control of skin function: the skin as a neuroimmunoendocrine organ. Physiol Rev. 2006;86(4):1309-1379.

60. Hans G, Deseure K, Adriaensen H. Endothelin-1-induced pain and hyperalgesia: a review of pathophysiology, clinical manifestations and future ther- apeutic options. Neuropeptides. 2008;42(2):119-132. 61. Yamamura H, Nabe T, Kohno S, Ohata K. Endothelin-1 induces release of histamine and leukotriene C4 from mouse bone marrow-derived mast cells. Eur J Pharmacol. 1994;257(3):235-242.

62. Yamamura H, Nabe T, Kohno S, Ohata K. Endothelin-1, one of the most potent histamine releasers in mouse peritoneal mast cells. Eur J Pharmacol. 1994;265(1-2):9-15.

63. Hultner L, Ehrenreich H. Mast cells and endothelin-1: a life-saving biological liaison? Trends Immunol. 2005;26(5):235-238.

64. Stassen M, Hultner L, Schmitt E. Classical and alternative pathways of mast cell activation. Crit Rev Immunol. 2002;22(2):115-140.

65. Shim WS, et al. TRPV1 mediates histamine-induced itching via the activation of phospholipase A2 and 12-lipoxygenase. J Neurosci. 2007;27(9):2331-2337.

66. Cattaruzza F, Cottrell GS, Vaksman N, Bunnett NW. Endothelin-converting enzyme 1 promotes resensitization of neurokinin 1 receptor-dependent neurogenic inflammation. Br J Pharmacol. 2009; 156(5):730-739.

67. Todt I, et al. Neurotological and neuroanatomical changes in the connexin-26-related HID/KID syndrome. Audiol Neurootol. 2006;11(4):242-248.

68. Scholzen TE, et al. Neutral endopeptidase terminates substance P-induced inflammation in allergic contact dermatitis. J Immunol. 2001;166(2):1285-1291.

69. Steinhoff M, et al. Proteinase-activated receptors: transducers of proteinase-mediated signaling in inflammation and immune response. Endocr Rev. 2005;26(1):1-43.

70. Roosterman D, et al. Intracellular degradation of somatostatin-14 following somatostatin-receptor3-mediated endocytosis in rat insulinoma cells. FEBS J. 2008;275(19):4728-4739.

71. Vohra BP, Planer W, Armon J, Fu M, Jain S, Heuckeroth RO. Reduced endothelin converting enzyme-1 and endothelin-3 mRNA in the developing bowel of male mice may increase expressivity and penetrance of Hirschsprung disease-like distal intestinal aganglionosis. Dev Dyn. 2007;236(1):106-117.

72. Steinhoff M, et al. Agonists of proteinase-activated receptor 2 induce inflammation by a neurogenic mechanism. Nat Med. 2000;6(2):151-158.

73. Pelayo JC, Poole DP, Steinhoff M, Cottrell GS, Bunnett NW. Endothelin-converting enzyme-1 regulates trafficking and signalling of the neurokinin 1 receptor in endosomes of myenteric neurones. J Physiol. 2011;589(pt 21):5213-5230.

74. Murphy JE, et al. Protein phosphatase 2A mediates resensitization of the neurokinin 1 receptor. Am J Physiol Cell Physio. 2011;301(4):C780-C791.

75. Hosogi M, Schmelz M, Miyachi Y, Ikoma A. Bradykinin is a potent pruritogen in atopic dermatitis: a switch from pain to itch. Pain. 2006; 126(1-3):16-23. 